\title{
Prefrontal Projections to the Thalamic Reticular Nucleus form a Unique Circuit for Attentional Mechanisms
}

\author{
Basilis Zikopoulos $^{1}$ and Helen Barbas ${ }^{1,2,3,4}$ \\ ${ }^{1}$ Department of Health Sciences and ${ }^{2}$ Program in Neuroscience, Boston University, Boston, Massachusetts 02215, ${ }^{3}$ New England Primate Research Center, \\ Harvard Medical School, Boston, Massachusetts 02115, and ${ }^{4}$ Department of Anatomy and Neurobiology, Boston University School of Medicine, Boston, \\ Massachusetts 02118
}

The inhibitory thalamic reticular nucleus (TRN) intercepts and modulates all corticothalamic and thalamocortical communications. Previous studies showed that projections from sensory and motor cortices originate in layer VI and terminate as small boutons in central and caudal TRN. Here we show that prefrontal projections to TRN in rhesus monkeys have a different topographic organization and mode of termination. Prefrontal cortices projected mainly to the anterior TRN, at sites connected with the mediodorsal, ventral anterior, and anterior medial thalamic nuclei. However, projections from areas 46, 13, and 9 terminated widely in TRN and colocalized caudally with projections from temporal auditory, visual, and polymodal association cortices. Population analysis and serial EM reconstruction revealed two distinct classes of corticoreticular terminals synapsing with GABA/parvalbumin-positive dendritic shafts of TRN neurons. Most labeled boutons from prefrontal axons were small, but a second class of large boutons was also prominent. This is in contrast to the homogeneous small TRN terminations from sensory cortices noted previously and in the present study, which are thought to arise exclusively from layer VI. The two bouton types were often observed on the same axon, suggesting that both prefrontal layers V and VI could project to TRN. The dual mode of termination suggests a more complex role of prefrontal input in the functional regulation of TRN and gating of thalamic output back to the cortex. The targeting of sensory tiers of TRN by specific prefrontal areas may underlie attentional regulation for the selection of relevant sensory signals and suppression of distractors.

Key words: laminar origin of projections; presynaptic specificity; macaque brain; dorsolateral prefrontal cortex; inhibitory control; 3D reconstruction

\section{Introduction}

The thalamic reticular nucleus (TRN) is composed entirely of inhibitory neurons, unlike other thalamic nuclei, and surrounds the thalamus along its entire anteroposterior extent. Corticothalamic and thalamocortical axons must, therefore, pass through its mesh of inhibitory neurons (supplemental Fig. 1, Movie 1, available at www.jneurosci.org as supplemental material) (Jones, 1985). The TRN receives unidirectional projections from the cortex and has bidirectional connections with all thalamic nuclei and is thus in a strategic position to modulate the flow of information between thalamus and cortex.

The TRN has a crude topographic map of the cortex and thalamus, so that cortices and their associated thalamic nuclei project to the same parts of TRN. This topography broadly divides TRN into motor and sensory sectors (for review, see Guillery et al., 1998). The topography, however, is blurred, hence

Received Dec. 22, 2005; revised April 21, 2006; accepted May 29, 2006.

This work was supported by National Institutes of Health grants from National Institute of Mental Health and National Institute of Neurological Disorders and Stroke. We thank Dr. Alan Peters for EM consultation, Dr. John Fiala for assistance in adapting the free 3D reconstruction software he developed, Dr. Ron Killiany for MRI and surgical assistance, Dr. Claus Hilgetag for helpful suggestions about the statistical analyses, Maria Medalla, Ola Alade, and Marcia Feinberg for technical assistance, Dr. P. Sehgal for veterinary care, and Linda Fernsten for surgical assistance.

Correspondence should be addressed to Helen Barbas, Boston University, 635 Commonwealth Avenue, Room 431, Boston, MA 02215. E-mail: barbas@bu.edu.

DOI:10.1523/JNEUROSCI.5511-05.2006

Copyright $\odot 2006$ Society for Neuroscience $\quad$ 0270-6474/06/267348-14\$15.00/0
TRN connection zones of nearby cortical or thalamic sites overlap, allowing indirect modulation of TRN inhibition.

The activity of TRN changes dramatically during the sleepwake cycle, shifting from spindle oscillations during slow-wave sleep that inhibit thalamocortical activity, to single-spike activity during wakefulness. These properties implicate TRN in attention, highlighted by TRN inhibition of thalamocortical neurons during cortically generated absence seizures accompanied by loss of consciousness (Pinault, 2004; Fuentealba and Steriade, 2005; Steriade, 2005). Because of its unique placement and physiology, it has been suggested that TRN is the attentional searchlight of the brain (Crick, 1984) and a key component of a larger network including the prefrontal cortex and thalamus, with an important role in cognition.

Despite these indications, there is no information on prefrontal corticoreticular projections in primates, the interaction of prefrontal areas, and their associated thalamic nuclei, or the pattern of prefrontal projections in TRN compared with other cortices. Sensory and motor cortices are thought to issue projections to TRN exclusively from layer VI, which terminate as small boutons (Guillery et al., 1998; Rouiller et al., 1998; Kakei et al., 2001). Layer $\mathrm{V}$ does not appear to contribute to the corticoreticular projection but innervates other thalamic nuclei through large boutons (Schwartz et al., 1991; Kakei et al., 2001). Corticothalamic projections from layer $\mathrm{V}$ are thought to be driving afferents 
of an intrathalamic corticocortical communication system, whereas layer VI projections are regarded as modulators of thalamic relay activity (Guillery, 1995).

The prefrontal system is an excellent model to investigate the relationship of high-order association areas with TRN because it issues substantial projections from layer $\mathrm{V}$ to other thalamic nuclei (Arikuni et al., 1983; Giguere and Goldman-Rakic, 1988; Xiao and Barbas, 2002, 2004). We examined the organization of projections from several prefrontal areas and their thalamic nuclei in TRN and compared them with projections to TRN from sensory and polymodal association cortices. Our results show, for the first time in a primate, the topography and mode of prefrontal corticoreticular terminations and establish that they differ from TRN projections originating in sensory cortices. Our findings provide the anatomic basis for the gating of sensory-related corticothalamic communications by the prefrontal cortex and TRN in mechanisms underlying selection or enhancement of relevant stimuli.

\section{Materials and Methods \\ Animal and tissue preparation}

Experiments were conducted on 12 adult rhesus monkeys (Macaca mulatta) in accordance with National Institutes of Health Guide for the Care and Use of Laboratory Animals (publication 80-22 revised, 1996). The experiments were approved by the Institutional Animal Care and Use Committee at Boston University School of Medicine, Harvard Medical School, and New England Primate Research Center. Procedures were designed to minimize animal suffering and reduce their number.

Magnetic resonance imaging. Before injection of neural tracers, we obtained three-dimensional (3D) scans of each brain using magnetic resonance imaging (MRI) in animals anesthetized with propofol (loading dose, $2.5-5 \mathrm{mg} / \mathrm{kg}$, i.v.; continuous rate infusion, $0.25-0.4$ $\left.\mathrm{mg} \cdot \mathrm{kg}^{-1} \cdot \mathrm{min}^{-1}\right)$. The interaural line was rendered visible in the scan by filling the hollow ear bars of the stereotaxic apparatus with betadine salve. We then calculated the stereotaxic coordinates for each injection in three dimensions using the interaural line as reference.

Surgical procedures. One week after the MRI, the monkeys were anesthetized with ketamine hydrochloride $(10-15 \mathrm{mg} / \mathrm{kg}$, i.m.) followed by gas anesthetic (isoflurane), until a surgical level of anesthesia was accomplished. Surgery was performed under aseptic conditions while heart rate, muscle tone, respiration, and pupillary dilatation were closely monitored. The monkeys were placed in a stereotaxic apparatus, and a small region of the cortex was exposed.

Injection of neural tracers. In an effort to minimize animal numbers, injections of distinct neural tracers were made in the right and left hemisphere, as indicated in Table 1 (for map of injection sites, see Fig. 1A-F). We injected tracers in prefrontal areas $9,10,46,13$, and 32, as well as temporal areas TE1, Ts1, Ts2, and 36, and the anteromedial (AM), ventral anterior (VA), and mediodorsal (MD) thalamic nuclei, which are connected with prefrontal cortices. Using a microsyringe (Hamilton, 5 $\mu \mathrm{l})$ mounted on a microdrive, we injected the bidirectional tracers biotinylated dextran amine (BDA) (10\% solution, $6-10 \mu \mathrm{l}, 10 \mathrm{kDa}$; Invitrogen, Carlsbad, CA), fluoro-emerald (FE) (dextran fluorescein, 10\% solution, $3-5 \mu \mathrm{l}, 10 \mathrm{kDa}$, and in some cases a mixture of 3 and $10 \mathrm{kDa}$; Invitrogen), fluoro-ruby (FR) (dextran tetramethylrhodamine, 10\% solution, 3-4 $\mu \mathrm{l}, 10 \mathrm{kDa}$, and in some cases a mixture of 3 and $10 \mathrm{kDa}$; Invitrogen), and Lucifer yellow (LY) (dextran, Lucifer yellow, anionic, lysine fixable, $10 \%$ solution, 3-4 $\mu \mathrm{l}, 10 \mathrm{kDa}$; Invitrogen). All of these tracers are excellent anterograde tracers that label the entire extent of axonal terminals and boutons. They also act as retrograde tracers (especially in the $3 \mathrm{kDa}$ molecular weight), but the extent of the labeling is restricted to the cell bodies and proximal dendrites of projection neurons and the tracer does not enter axon collaterals (Veenman et al., 1992; Richmond et al., 1994; Reiner et al., 2000). In some cases, we injected the retrograde tracer fast blue $(\mathrm{FB})$ in the thalamus (MD and VA; $1 \%$ solution, volume of $0.3 \mu \mathrm{l}, 379.84 \mathrm{Da}$; Sigma, St. Louis, MO). Trans-synaptic transport of any of these tracers has not been observed. In each case, the
Table 1. Injection sites and neural tracers used in $\mathbf{1 2}$ cases analyzed

\begin{tabular}{llll}
\hline Case name & Injection site & Brain hemisphere & Tracer \\
\hline AT & 36 & Right & BDA \\
AV & TE1/Ts1 & Left & BDA \\
AY & 32 & Left & BDA \\
BA & 10 & Right & BDA \\
& TE & Left & FR \\
BB & MD & Left & FE \\
& AM & Left & FR \\
BC & 10 & Left & BDA \\
& 13 & Right & FE \\
& Ts2 & Right & FR \\
BD & VA & Left & FE \\
& AM & Left & FR \\
& MD & Left & FB \\
BE & VA & Left & FR \\
& VA & Left & FB \\
BF & 10 & Left & BDA \\
BG & 9 & Left & BDA \\
& 32 & Right & BDA \\
& VA & Right & FE \\
& VA & Right & FR \\
& MD & Right & FB \\
BH & Dorsal 46 & Left & BDA \\
& 9 & Right & FR \\
& 13 & Right & LY \\
BI & 32 & Right & BDA \\
& Dorsal 46 & Left & FR \\
& 13 & Left & \\
\hline
\end{tabular}

tracer was injected in two penetrations and, for the cortical injections, at a depth of 1.2-1.6 mm below the pial surface. After injection, the needle was left in place for $10-15$ min to allow the dye to penetrate at the injection site and prevent upward diffusion of the dye during retraction of the needle.

Perfusion and tissue processing. After a survival period of $18 \mathrm{~d}$, the animals were anesthetized with a lethal dose of sodium pentobarbital (>50 mg/kg, to effect) and transcardially perfused with $4 \%$ paraformaldehyde or, for electron microscopy, $4 \%$ paraformaldehyde and $0.2 \%$ glutaraldehyde in $0.1 \mathrm{~m}$ phosphate buffer (PB), $\mathrm{pH}$ 7.4. The brains were removed from the skull, photographed, and cryoprotected in graded solutions of sucrose $(10-30 \%)$ in $0.01 \mathrm{M}$ PBS. Brains were then frozen in $-75^{\circ} \mathrm{C}$ isopentane (Fisher Scientific, Pittsburgh, PA) and cut on a freezing microtome in the coronal plane at 40 or $50 \mu \mathrm{m}$ to produce 10 series. In cases with injection of fluorescent tracers, one series was mounted on glass slides, coverslipped, and used to map labeled neurons and terminals.

\section{Histochemical and immunohistochemical procedures}

Bright-field or fluorescent microscopy. In cases with BDA injections, freefloating sections were rinsed in $0.01 \mathrm{M}$ PBS and incubated for $1 \mathrm{~h}$ in an avidin-biotin HRP complex [AB kit (Vector Laboratories, Burlingame, CA); diluted $1: 100$ in $0.01 \mathrm{M}$ PBS with $0.1 \%$ Triton X-100]. The sections were then washed and processed for immunoperoxidase reaction using diaminobenzidine (DAB) (Zymed, San Francisco, CA). Sections were then mounted, dried, and coverslipped with Permount or Entellan (Merck, Whitehouse, NJ).

In some cases with FE, FR, and LY injections in the prefrontal cortex, we used polyclonal antibodies (anti-fluorescein/Oregon Green, antitetramethylrhodamine, and anti-LY, 1:800; Invitrogen) to convert the fluorescent tracers and visualize them by the peroxidase-catalyzed polymerization of DAB. To avoid cross-reaction with the BDA, we used nonbiotinylated polyclonal anti-rabbit secondary antibody followed by tertiary anti-rabbit solution (PAP method, 1:200; Sternberger Monoclonals, Lutherville, MD).

To identify TRN inhibitory neurons, we used antibodies against the inhibitory neurotransmitter GABA or the calcium binding protein parvalbumin (PV). The tissue was rinsed in $0.01 \mathrm{~m} \mathrm{PBS,} \mathrm{pH} \mathrm{7.4,} \mathrm{followed} \mathrm{by}$ 
$10 \%$ normal goat serum, $5 \%$ bovine serum albumin, and $0.1 \%$ Triton $\mathrm{X}-100$ in $0.01 \mathrm{~m}$ PBS blocking solution for $1 \mathrm{~h}$ and incubated for $1-2 \mathrm{~d}$ in primary antibody for GABA [1:1000, mouse monoclonal (Sigma) or 1:1000, rabbit polyclonal (DiaSorin, Stillwater, MN] or PV (1:2000, mouse monoclonal; Chemicon, Temecula, CA). The sections were rinsed in PBS, incubated for $1 \mathrm{~h}$ with a goat anti-rabbit (for GABA polyclonal) or anti-mouse (for GABA and PV monoclonals) IgG conjugated with the fluorescent probes Alexa Fluor 488 (green) or 568 (red; 1:100; Invitrogen) and thoroughly rinsed with PBS. In some cases for PV labeling, a biotinylated secondary antibody and an avidin-biotin-peroxidase kit was used to label PV-positive $\left(\mathrm{PV}^{+}\right)$structures with $\mathrm{DAB}$. For the quantification of TRN neurons, cells were labeled with an antibody against the DNA-binding, neuron-specific nuclear protein NeuN (1:200, mouse monoclonal; Chemicon). To exclude any nonspecific immunoreactivity, control experiments were performed, with sections adjacent to those used in the experiments described above. These included omission of the primary antibodies and incubation with all secondary or tertiary antisera used. All control experiments resulted in no immunocytochemical labeling.

Electron microscopy. Sections treated for bright-field BDA, FE, FR, or LY were processed for electron microscopy. We used the protocols described above, but Triton X-100 concentration in all solutions was reduced to $0.025 \%$. To determine the relationship of labeled boutons from prefrontal areas with inhibitory TRN neurons, we double labeled sections using antibodies against GABA or PV and preembedding immunohistochemistry and visualized label with gold-conjugated anti-mouse or anti-rabbit secondary antibodies (1:50; gold particle diameter, $1 \mathrm{~nm}$; Amersham Biosciences, Piscataway, NJ). To prevent diffusion of the gold particles, tissue was postfixed in a microwave with $2 \%$ glutaraldehyde (described by Jensen and Harris, 1989). Labeling was intensified with the use of the Amersham Biosciences silver enhancement kit (IntenSE). Tissue sections were mounted on slides and quickly viewed under the light microscope, and images were captured with a CCD camera. Small blocks of sections with anterograde and GABA or PV label were cut under a dissecting microscope, postfixed in $1 \%$ osmium tetroxide with $1.5 \%$ potassium ferrocyanide in $\mathrm{PB}$, washed in buffer $(\mathrm{PB})$ and water, and dehydrated in an ascending series of alcohols. While in $70 \%$ alcohol, they were stained with $1 \%$ uranyl acetate for $30 \mathrm{~min}$. Subsequently, they were cleared in propylene oxide and embedded in araldite at $60^{\circ} \mathrm{C}$. Serial ultrathin sections $(50 \mathrm{~nm})$ were cut with a diamond knife (Diatome, Fort Washington, PA) using an ultramicrotome (Ultracut; Leica, Wein, Austria) and collected on single slot grids, to view with a transmission electron microscope (100CX; Jeol, Peabody, MA).

\section{Stereological analysis}

The architecture of thalamic nuclei, the cortex, and other brain areas was determined in Nissl and AChE-stained sections. The nomenclature of the thalamus is according to the map of Jones (1985), as modified from the map of Olszewski (1952), and for the prefrontal cortex is according to the map of Barbas and Pandya (1989), modified from the map of Walker (1940).

Bouton population analysis. We analyzed anterograde labeling in the TRN at high magnification $(1000 \times)$ using unbiased stereological methods (for review, see Howard and Reed, 1998). Progressive mean analysis was used to determine the systematic, random sampling fraction, which was $1 / 100$ of the total volume of the region studied and resulted in measuring the morphological characteristics of $>4000$ labeled bouton profiles in each case. In three cases with tracer injections in temporal cortices (case BA, area TE; case BC, area Ts2; and case AT, area 36), we sampled terminations in TRN exhaustively to map the distribution of labeled boutons and measure their size. The morphological characteristics measured were the minor and major diameter, perimeter, and surface area.

Neuron and bouton density estimation. We estimated the numbers of $\mathrm{NeuN}^{+}$neurons, $\mathrm{PV}^{+}$neurons, and anterogradely labeled boutons in TRN using the unbiased stereological method of the optical fractionator (Gundersen, 1986; Howard and Reed, 1998) with the aid of a commercial system (StereoInvestigator; MicroBrightField, Williston, VT). Large and small boutons were measured separately and systematically distinguished based on the results of the bouton population analyses presented earlier. Specifically, in agreement with previous studies (Schwartz et al.,
1991; Ojima et al., 1996; Rouiller et al., 1998), small boutons had a maximum major diameter of $\sim 1.4 \mu \mathrm{m}$, and, therefore, all bigger terminals were considered as large. We used progressive mean analysis and a pilot study to determine the sampling fraction, which was $1 / 160$ of the total volume of TRN and was achieved by setting the disector counting frame at $100 \times 100 \mu \mathrm{m}$ and the grid size at $400 \times 400 \mu \mathrm{m}$. The total number of neurons or boutons counted in the systematic random samples was multiplied with the denominator of the sampling fraction to provide an estimate of the total number of labeled neurons or boutons. The volume of TRN was estimated by multiplying the number of measured disectors with the same denominator and was used to assess the density of labeled boutons in each case by dividing the total number of boutons by the corresponding TRN volume. We normalized data by expressing the density of neurons or large and small boutons as a percentage of the total density of all neurons or boutons in each case.

\section{$3 D$ reconstruction and imaging}

Light microscopy. In some cases, the labeled axonal pathways were followed all the way from the injection sites in the prefrontal cortex to their termination sites in TRN, at low magnification $(40 \times)$ in serial sections. The section surface, the cytoarchitectonic borders of various nuclei, and labeled pathways, were outlined with the aid of a commercial computerized microscope system and motorized stage (Neurolucida; MicroBrightField).

Sections ipsilateral to the injection sites containing areas of interest (TRN, MD, VA, caudate, and amygdala) were viewed under bright-field or fluorescence illumination, and their surface as well as architectonic borders were outlined using the Neurolucida software. The procedure involves setting a reference point for every brain hemisphere analyzed, and, as a result, the outlines are automatically registered and aligned to the actual corresponding sections, retaining information about the 3D coordinates of every mark or trace. These sections and outlines were used for stereological analyses of the anterograde labeling presented earlier. Retrogradely labeled neurons in the TRN directed to MD, VA, and AM were mapped and their stereotaxic coordinates were marked.

To compare the topography and distribution of anterograde and retrograde labeling across cases and exhibit their overlap in TRN, we reconstructed in three dimensions the entire nucleus using the free, open source software Reconstruct (Fiala, 2005). With the aid of Neurolucida, we outlined the surface and the borders of TRN of all Nissl-stained coronal sections spanning the rostrocaudal extent of the nucleus from a representative case and used them as reference. We then imported these reference outlines along with outlines containing 3D information about the topography of labeling in Reconstruct, aligned them, and generated $3 \mathrm{D}$ models. As a result, all markers and traces were stereotaxically registered and superimposed on the 3D models. This method made it possible to compare the relative distribution of different cortical axonal terminations in TRN and labeled neurons in TRN projecting to MD, VA, or AM to assess possible overlaps.

To exhibit the distribution of large and small terminals in TRN originating in different prefrontal areas, we imported Neurolucida outlines of sections and labeling in Reconstruct, in which we color coded the bouton markers (large boutons, blue; small boutons, red) and generated 3D maps.

Images presented or used for analyses were captured at high resolution with a CCD camera mounted on an Olympus Optical (Thornwood, NY) BX51 microscope connected to a personal computer using commercial imaging systems [MetaMorph version 4.1 (Universal Imaging Corporation) or Neurolucida]. Image stacks of several focal planes were acquired in each area of interest, resulting in pictures with high depth of field of 40to $50-\mu \mathrm{m}$-thick sections focused throughout their $z$-axis extent. Stacked images from adjacent serial sections were superimposed to highlight the differential distribution or overlap of retrogradely labeled neurons and anterogradely labeled boutons. Photomicrographs were prepared with Adobe Photoshop (Adobe Systems, San Jose, CA), and overall brightness and contrast were adjusted without retouching.

Stereological measurements of the numbers of labeled fibers that contained exclusively small or large boutons or a mixed population were conducted on high-resolution stacks of images captured as described above. These stacks were imported in NIH ImageJ, reconstructed in 3D, 
and rotated, in $1^{\circ}$ increments in the $x$-, $y$-, or $z$-axis, using the $3 \mathrm{D}$ project plugin (supplemental Movie 2, available at www.jneurosci.org as supplemental material). This allowed us to distinguish whether various boutons were part of the same or different labeled axons.

Electron microscopy. We viewed labeled boutons emanating from prefrontal axons synapsing on TRN neurons and neighboring unlabeled boutons at high magnification $(10,000 \times)$ and photographed them throughout their whole extent (between 10 and 80 serial ultrathin sections). Film negatives were scanned at high resolution (Minolta Dimage Scan Multi II; Konica Minolta, Mahwah, NJ) and imported as a series in Reconstruct, in which they were aligned. Boutons, postsynaptic densities, and postsynaptic structures (e.g., dendrites) were traced. Section thickness was calibrated through measurements of the diameter of mitochondria (Fiala and Harris, 2001). Structures were reconstructed in three dimensions, and their volumes, surface areas, and diameters were calculated. We used classic criteria for identifying synapses and profiles (for review, see Peters et al., 1991) as described previously (Barbas et al., 2003; Germuska et al., 2006).

\section{Statistical analysis}

Data were evaluated with Statistica (StatSoft, Tulsa, OK), through scatter and frequency distribution plots and $K$-means cluster analysis with parameters set to maximize initial between-cluster distances. $\chi^{2}$ and Kolmogorov-Smirnov tests were used to examine the bouton size distributions. We used ANOVA to test for differences among bouton populations and densities, and $p$ values $<0.01$ were taken as statistically significant. Post hoc analyses using Bonferroni's/Dunn's (all means) was performed to identify possible differences between groups.

\section{Results \\ TRN features}

The TRN covers the dorsal and lateral parts of the thalamus as an open umbrella with the handle positioned on the midline (supplemental Figs. 1, 2, Movie 1, available at www.jneurosci.org as supplemental material). It has a similar shape across animals, appearing round rostrally, becomes progressively thinner along the mediolateral dimension and elongates along the dorsoventral axis, and tapers to a narrow spur at its most caudal extent. The TRN in the rhesus monkey was remarkably similar across cases, containing $28 \pm 2$ sections (mean $\pm \mathrm{SE}$ ), with a mean volume of $44 \pm 3.7 \mathrm{~mm}^{3}$. On each side, the TRN consisted of $\sim 1$ million neurons $(1,077,091 \pm 108,509)$ and a mean density of 15,093 \pm 73 neurons $/ \mathrm{mm}^{3}$. All neurons in TRN were GABAergic and most were $\mathrm{PV}^{+}(\sim 70 \pm 13 \%$; mean density $\pm \mathrm{SE}, 10,588 \pm 1,862$ neurons $/ \mathrm{mm}^{3}$ ) (supplemental Fig. 1, available at www.jneurosci. org as supplemental material). The small variability in TRN size and shape facilitated a reliable comparison of projections from cortical areas across cases through $3 \mathrm{D}$ reconstruction.

To facilitate the description of projections and overlaps in TRN, the nucleus was divided rostrocaudally into four quarters. The rostral quarter extended from the rostral pole of TRN to the anterior extent of the VA and the anterior thalamic nuclei. The rostral pole was bordered laterally by the internal capsule and medially by the bed nucleus of the stria terminalis. The central quarters of TRN, namely the rostrocentral and caudocentral, were crescent shaped and surrounded the central VA, MD, lateral posterior, ventral nuclei, and the anterior part of the pulvinar. The caudal quarter of TRN surrounded the rest of the pulvinar and included the perigeniculate area.

\section{Topography of prefrontal terminations in the thalamic reticular nucleus}

The injection sites of anterograde tracers in prefrontal cortices are shown on the lateral (Fig. $1 A$ ), medial (Fig. $1 B$ ), and basal (Fig. 1C) surfaces of the brain. Three-dimensional reconstruction of the injection sites showed that injections encompassed all cortical layers, and the needle tracts were restricted to the cortical mantle. We mapped the distribution of labeled terminals from cortical axons in TRN in one series of sections ( $1 / 10$ of the total nucleus) and estimated stereologically the axonal bouton density.

In all cases, labeled axons from prefrontal cortices entered TRN rostrally from the adjacent internal capsule and some axons passed through TRN without sending terminations (Fig. 2A). A majority of prefrontal corticoreticular terminals were found in primarily overlapping sites of the anterior and dorsal TRN. On average, $83 \%$ of labeled boutons were distributed in the rostral quarter of TRN. The central quarters of TRN included, respectively, 6 and 9\%, and the caudal quarter of the nucleus had a few labeled boutons only after injections in area $9(\sim 2 \%)$.

Axons from area 32 terminated predominantly in the first quarter of the nucleus ( $\sim 98 \%)$, concentrated mainly in the rostral pole, with only $2 \%$ in the second quarter of TRN. Although anterograde labeling was prominent in the dorsal part of TRN, a few terminals were scattered along its ventral and central regions (Fig. 2B). A similar distribution of anterograde label was observed after injections in area 10 in the frontal pole. Labeled terminals were found only in the first quarter of TRN and almost exclusively in its dorsal part (Fig. 2B).

In contrast to the above areas, axons from the dorsomedial part of area 9 terminated in patches throughout the nucleus (Fig. $2 B)$. Labeled boutons were found predominantly in the dorsal part of TRN, most within the first quarter $(\sim 86 \%)$, but some were seen also in the central and caudal quarters of the nucleus $(\sim 14 \%)$. Axonal terminations from dorsal area 46 occupied larger zones of the first three quarters of TRN $(\sim 69,14$, and $17 \%$, respectively) and extended throughout the dorsoventral axis of the nucleus. Among prefrontal areas studied, dorsal area 46 issued the most robust projections to central-caudal regions of TRN (second and third quarters of the nucleus) (Fig. $2 B$ ). The same pattern, but with sparser label, was seen in the axonal terminations of orbital area 13, which were found in the first three quarters of TRN ( $\sim 66,9$, and $25 \%$, respectively) and spread over dorsal and ventral sites of the nucleus (Fig. $2 B$ ).

\section{Projections from areas 46, 13 , and 9 colocalized with cortical auditory, visual, and polymodal terminations in TRN}

We then investigated whether these posterior terminations of prefrontal axons overlapped with projections from temporal auditory (Ts1, Ts2), visual (TE1), and polymodal (36) association areas in four cases (case BC, area Ts2; case AT, area 36; case BA, area TE1; and case AV, areas Ts1/TE1) (Table 1; Fig. $1 A, C)$. The comparison was made from different experiments after alignment and registration of all sections containing TRN. Labeled terminals were superimposed on a reference map, and threedimensional reconstruction revealed a significant extent of colocalization (Fig. 3). Anterogradely labeled terminals in the TRN from Ts1 and Ts2 were found mainly in its caudal regions, consistent with the location of the auditory sectors of TRN in other species. Axonal terminations from areas 36 and TE1 were found rostral to the auditory sector, in the visual sector of TRN, situated in the central-caudal parts of the nucleus. These projections were more prominent in the ventral parts of the nucleus, which included the perigeniculate region. Interestingly, among prefrontal areas studied only terminations from dorsal area 46 overlapped with projections from all of the injected temporal cortices in the caudocentral and ventral segments of TRN, in the sensory sectors of the nucleus (Fig. 3). Projections from orbital area 13 and temporal areas 36 and Ts2 overlapped extensively in dorsal and ventral parts of the sensory TRN sectors. In contrast, there was only 

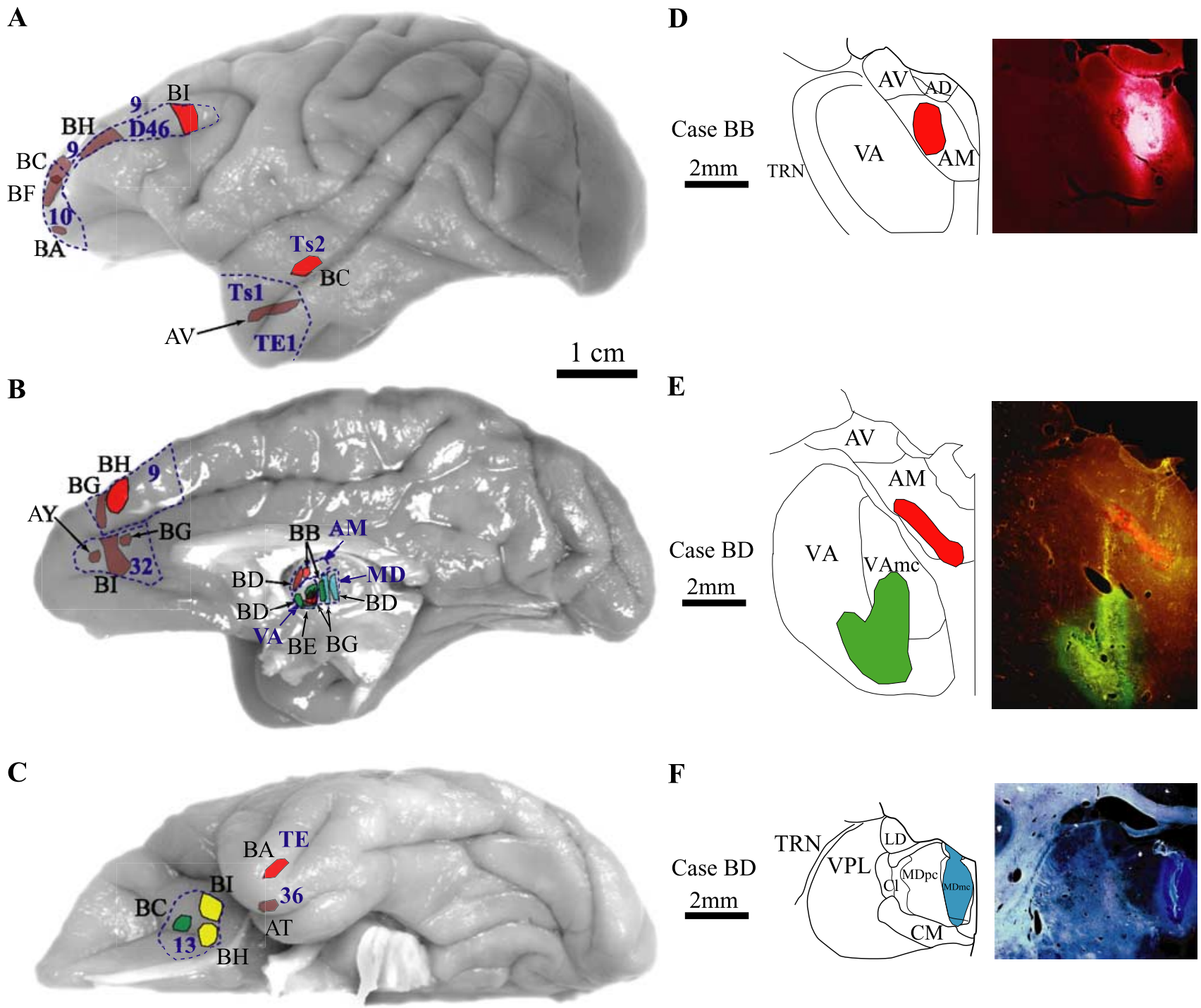

Figure 1. Composite of cortical and thalamic injection sites. Cortical injection sites shown on the lateral $(\boldsymbol{A})$, medial $(\boldsymbol{B})$, and basal $(\boldsymbol{C})$ surfaces of the brain of the rhesus monkey. $\boldsymbol{D}-\boldsymbol{F}$, Representative coronal sections through the thalamus (photomicrographs on the right column and corresponding outlines on the left column). Brown, BDA; red, fluoro-ruby; green, fluoro-emerald; yellow, Lucifer yellow; blue, fast blue. Case names appear in black. Outlines and names of areas and nuclei in A-Care in blue. AD, Nucleus anterior dorsalis; AV, nucleus anterior ventralis; Cl, nucleus centralis lateralis; CM, nucleus centrum medianum; LD, nucleus lateralis dorsalis; MDmc, nucleus medialis dorsalis, pars magnocellularis; MDpc, nucleus medialis dorsalis, pars parvicellularis; TRN, thalamic reticular nucleus; VAmc, nucleus ventralis anterior, pars magnocellularis; VPL, nucleus ventralis posterior lateralis.

limited overlap in the terminations from area 9 with those from areas 36 and Ts2, restricted to the dorsal regions of TRN (Fig. 3B).

\section{Prefrontal terminations colocalized with TRN connections with MD, VA, and AM thalamic nuclei}

We then investigated whether axonal terminations from prefrontal cortices were localized in sites of TRN that are connected with thalamic nuclei associated with prefrontal cortices. We focused on $\mathrm{MD}, \mathrm{VA}$, and $\mathrm{AM}$, three thalamic nuclei that receive the majority of prefrontal cortical input (Dermon and Barbas, 1994; Kultas-Ilinsky et al., 1995; Tai et al., 1995; Ilinsky et al., 1999; Xiao and Barbas, 2004) (for review, see Steriade et al., 1997). After injection of retrograde tracers in each of these thalamic nuclei (Fig. 1B,D-F), labeled projection neurons in TRN were most prevalent in the anterior and dorsal half of the nucleus, but some were also found in its ventral sector. Both VA and AM received projections from rostral TRN, especially its medial segment, whereas projection neurons directed to $\mathrm{MD}$ originated from a wider region, encompassing the first three quarters of TRN.
Reconstruction of TRN in three dimensions and overlay of terminals from prefrontal cortices and retrogradely labeled neurons that projected to $\mathrm{AM}, \mathrm{VA}$, and $\mathrm{MD}$ revealed that prefrontal axons terminated primarily in TRN sectors that are reciprocally connected with AM (Fig. 4A), VA (Fig. 4B), and MD (Fig. 4C). It is evident that there is a linkage throughout nearly the entire rostrocaudal extent of TRN with nuclei associated with the prefrontal cortex (Kievit and Kuypers, 1975, 1977). When these reconstructions included the anterograde terminals from auditory and visual association cortices TE1 and Ts1, a partial overlap with projections from dorsal area 46 was seen in the third quarter of $\mathrm{TRN}$, in which some reticular projection neurons to MD were also found (Fig. 5).

\section{Dual-termination mode of prefrontal axons in TRN}

Corticothalamic terminals were both of the en passant and terminal type and were polymorphic. At least two classes of labeled presynaptic profiles could be distinguished (Fig. 6A) (supplemental Movie 2, available at www.jneurosci.org as supplemental 

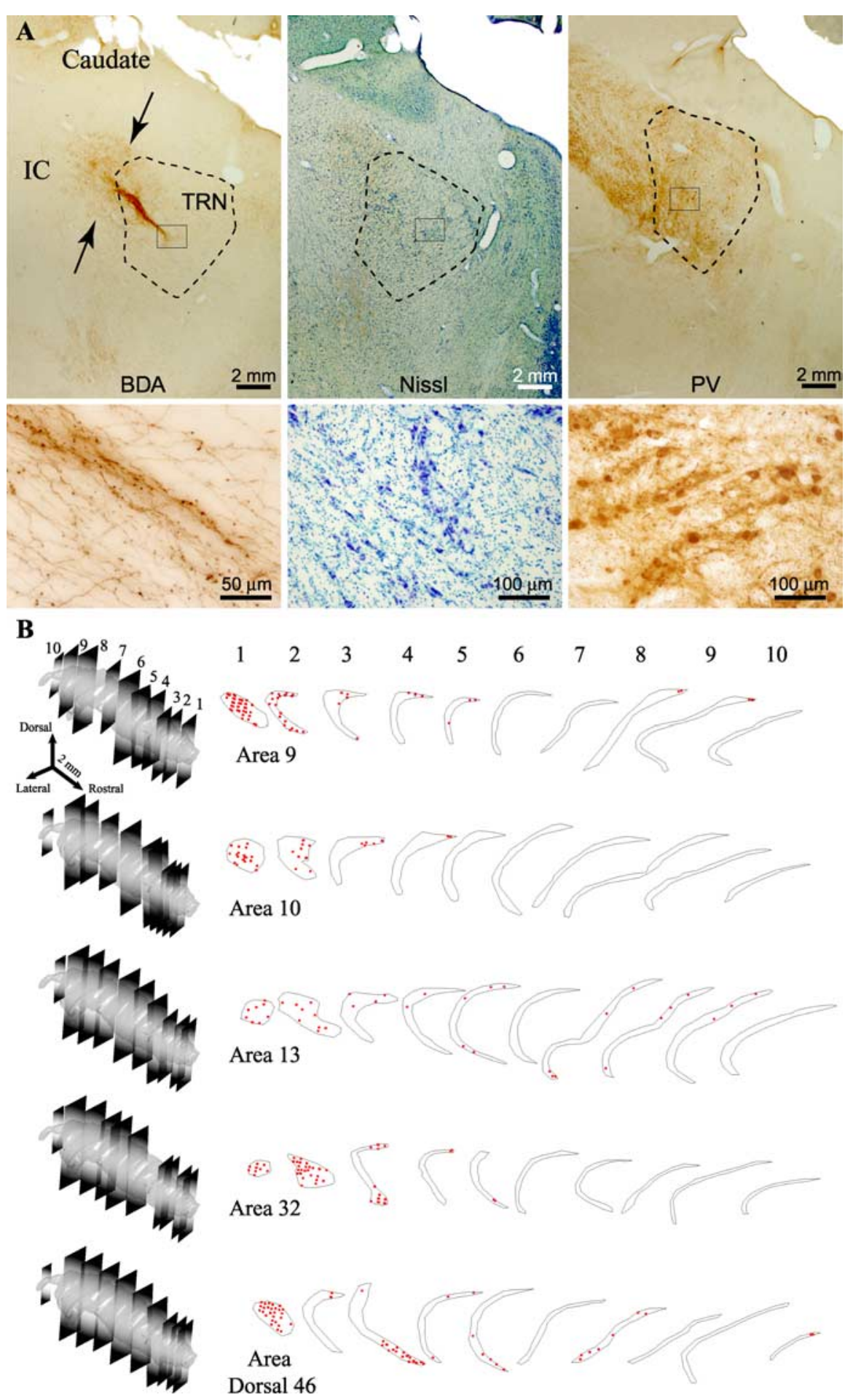

Figure 2. Prefrontal axonal terminations in the reticular nucleus. $\boldsymbol{A}$, Corticothalamic pathway from area 32 to TRN (arrows in left column), labeled with BDA, entering through the internal capsule (IC), shown at low magnification (top) and high magnification (bottom). The middle and right columns show matched sections at low (top) and high (bottom) magnification stained with Nissl (middle) or immunostained for PV (right) to show the borders of TRN. $\boldsymbol{B}$, Two-dimensional quantitative projection patterns in 10 representative coronal sections covering the whole rostrocaudal extent of TRN, shown in 3D reconstructions on the left. Each set of outlines corresponds to axonal boutons (red dots) from one prefrontal area. Each dot represents $\sim 10$ boutons. enabled us to construct a bouton population profile in TRN. Scatter and frequency distribution plots showed that the population of boutons was continuous, covering a wide range of sizes $(0.1 \mu \mathrm{m}<$ diameter $<4.5 \mu \mathrm{m}$ ) (Fig. $6 B$ ). However, cluster analysis indicated that there were at least two distinct bouton populations ( $p<$ 0.00001 ), one corresponding to the small terminals (mean diameter \pm variance, $0.82 \pm 0.05 \mu \mathrm{m}$ ), and the other corresponding to large boutons (mean diameter \pm variance, $1.69 \pm 0.19 \mu \mathrm{m})$. Moreover, comparison of all prefrontal projections revealed that all labeled bouton populations found in TRN were similar, regardless of origin in prefrontal cortex.

To quantitatively map the distribution and density of large and small terminals in TRN emanating from prefrontal cortices, we estimated their numbers using stereological procedures. Large boutons were on average $9 \%$ of the population (mean density, 1176 boutons $/ \mathrm{mm}^{3}$ ), and small boutons made up the other $91 \%$ (mean density, 15,015 boutons $/ \mathrm{mm}^{3}$ ). With the exception of cases with tracer injections in dorsal area 46 , the vast majority of large boutons were found in the rostrodorsal regions of TRN, occupying more focal sites than the small terminals, often overlapping with them (Fig. 7A). Large boutons observed in central and posterior sectors of TRN emanated exclusively from axons of dorsal area 46. In addition to the more widespread distribution of large boutons from dorsal area 46 , quantitative analysis revealed that they comprised $14 \%$ of all labeled terminals in TRN compared with $<10 \%$ from all other prefrontal areas (Fig. $7 B)$. Moreover, in central and posterior sectors of TRN, large and small boutons from dorsal area 46 rarely occupied overlapping sites (Fig. 7A).

Additional stereological analysis, 3D reconstruction, and rotation of $50-\mu \mathrm{m}$ thick sections (as described in Materials and Methods) revealed that the majority of labeled axons formed only small boutons ( $\sim 62 \%$ of all labeled axons with terminals in TRN), whereas only $9 \%$ of the axons gave rise exclusively to large boutons (Fig. 7C-E) (supplemental Movie 2, available at www.jneurosci.org as supplemental material). In a few occasions, large boutons branching out from the latter axmaterial). Most corticoreticular boutons were small (diameter $<1.4 \mu \mathrm{m}$ ) and spherical in shape, but a significant number of large $(1.4 \mu \mathrm{m}<$ diameter $<4.5 \mu \mathrm{m})$, round, ovoid, or irregularly shaped boutons were also present. Stereological sampling and measurement of the major diameter, perimeter, and crosssectional surface area of at least 4000 labeled profiles per case ons formed aggregated clusters, resembling bunches of grapes, as described for sensory cortical projections in several other thalamic nuclei but not TRN (Ojima, 1994; Bourassa et al., 1995; Ojima et al., 1996). Some axons with large en passant boutons formed round arbors at the termination sites, similar to those described for corticopulvinar projections (Rockland, 1996). The 

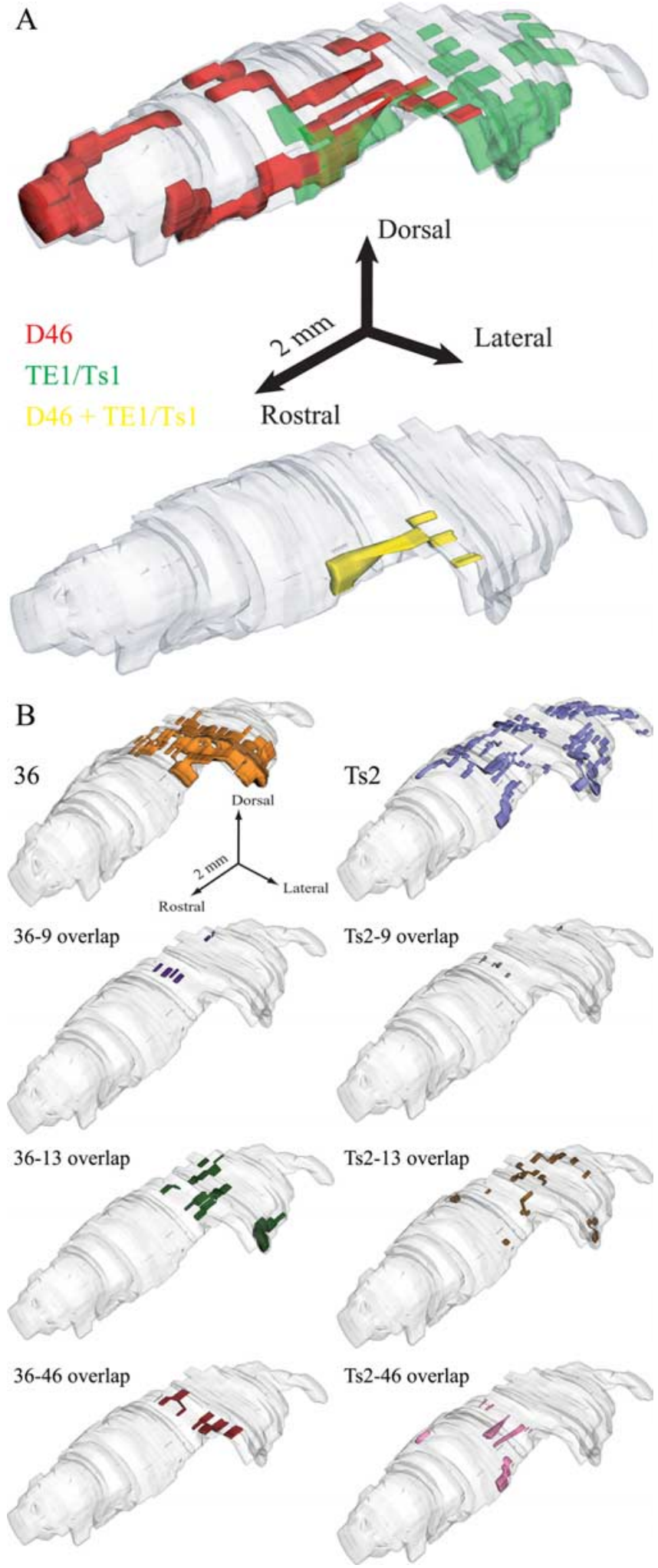

Figure 3. Prefrontal and temporal cortical terminations in the reticular nucleus reconstructed in three dimensions. A, Projections to TRN from dorsal area 46 (red) and TE1/Ts1 (green). Overlapping terminals can be seen at the bottom $3 \mathrm{D}$ model in yellow. $\boldsymbol{B}$, Projections to TRN from areas 36 (left column) and Ts2 (right column) and their respective overlaps with projections from prefrontal areas 9,13 , and 46 . The overlaps of area 9 with the temporal cortices studied are minor, whereas the overlaps of areas 13 and 46 with the same temporal cortices are more extensive.
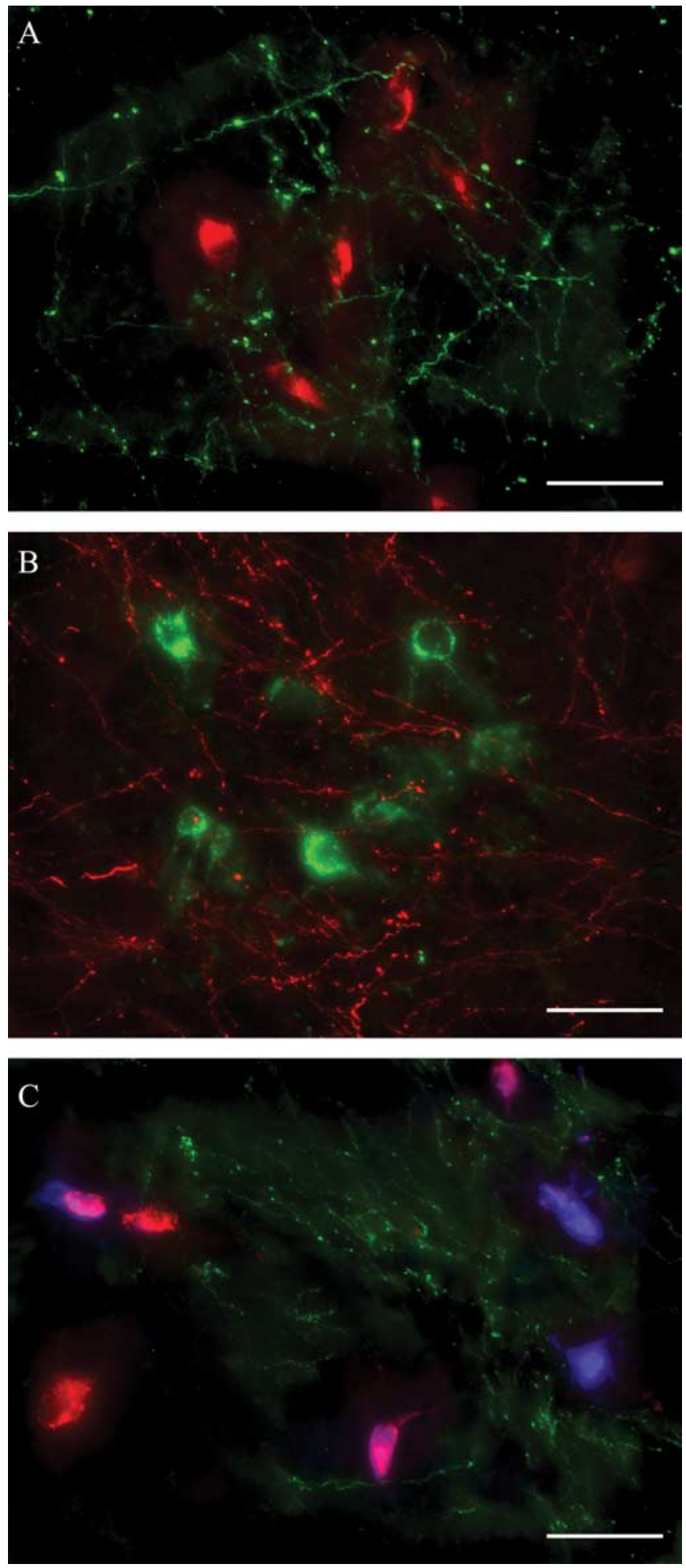

Figure 4. Colocalization of prefrontal axonal terminations with TRN projection neurons directed to anterior and medial thalamic nuclei. $\boldsymbol{A}$, Terminals from area 10 (green) overlap with labeled neurons in TRN projecting to AM (red). $\boldsymbol{B}$, Terminals from area 32 (red) overlap with labeled neurons in TRN projecting to VA (green). $\boldsymbol{C}$, Terminals from area 46 (green) overlap with labeled neurons in TRN projecting to VA (red and violet) and MD (blue and violet). Cells that appear violet are double labeled, projecting to MD and VA. Scale bars, $50 \mu \mathrm{m}$.

rest of the axonal branches (29\%) gave rise to a mixture of small and large boutons. Interestingly, although large boutons made up $\sim 9 \%$ of the prefrontal labeled terminals in TRN, they were observed on $38 \%$ of the labeled axonal branches. 
A
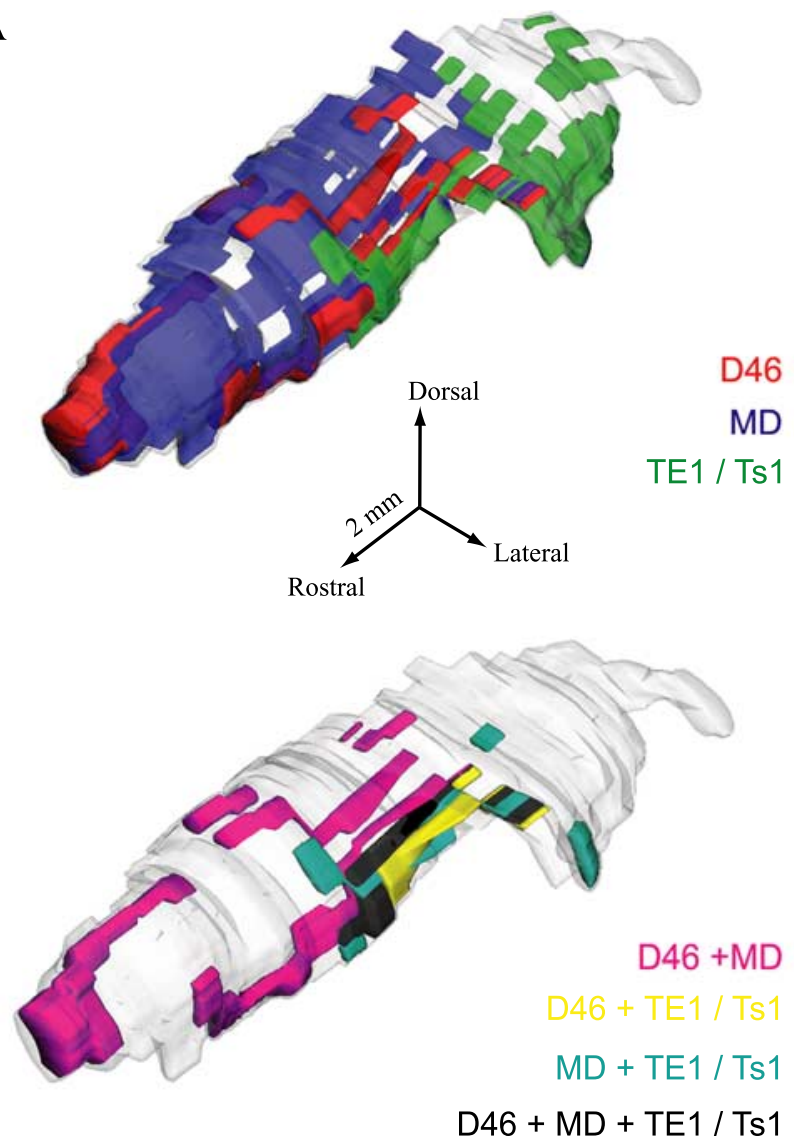

B

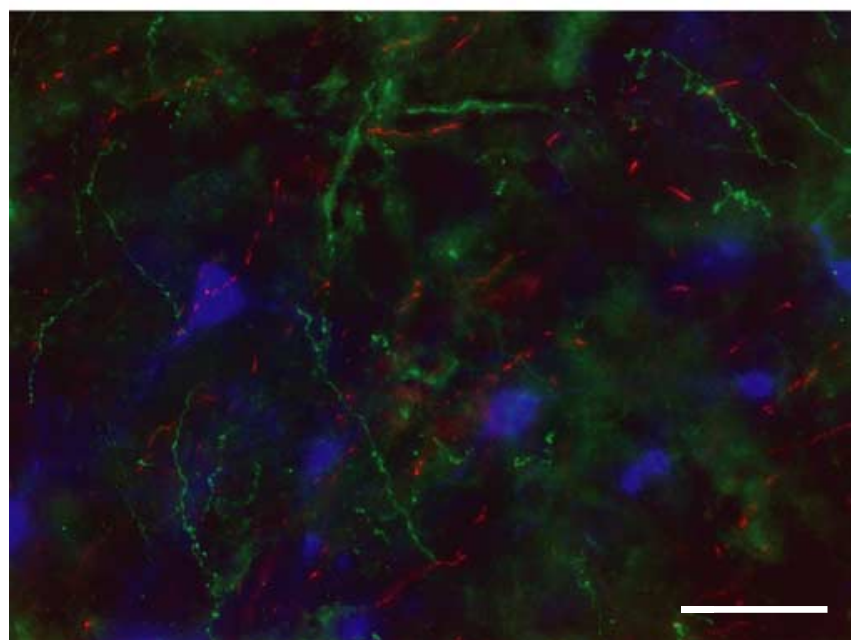

Figure 5. Widespread prefrontal terminations in TRN overlap with projections from sensory association cortices. $\boldsymbol{A}, 3 \mathrm{D}$ reconstruction of projections to TRN from areas 46 (red), TE1/Ts1 (green), and projection neurons to MD (blue). Overlapping terminals can be seen at the bottom 3D model in magenta (for dorsal area 46 and MD), yellow (for dorsal area 46 and TE1/Ts1), cyan (for MD and TE1/Ts1), and black (for dorsal 46, MD, and TE1/Ts1). B, Terminals from areas 46 (green) and TE1/Ts1 (red) overlap with labeled neurons in TRN projecting to MD (blue). Scale bar, $50 \mu \mathrm{m}$.

\section{Features of prefrontal corticoreticular synapses}

Ultrastructural analysis of serial ultrathin sections at the EM level and subsequent $3 \mathrm{D}$ reconstruction suggested the presence of at least two classes of labeled presynaptic profiles in TRN, both forming asymmetric (presumed excitatory) synapses with goldlabeled $\mathrm{PV}^{+}$or $\mathrm{GABA}^{+}$dendritic shafts (Figs. 8, 9). The first class

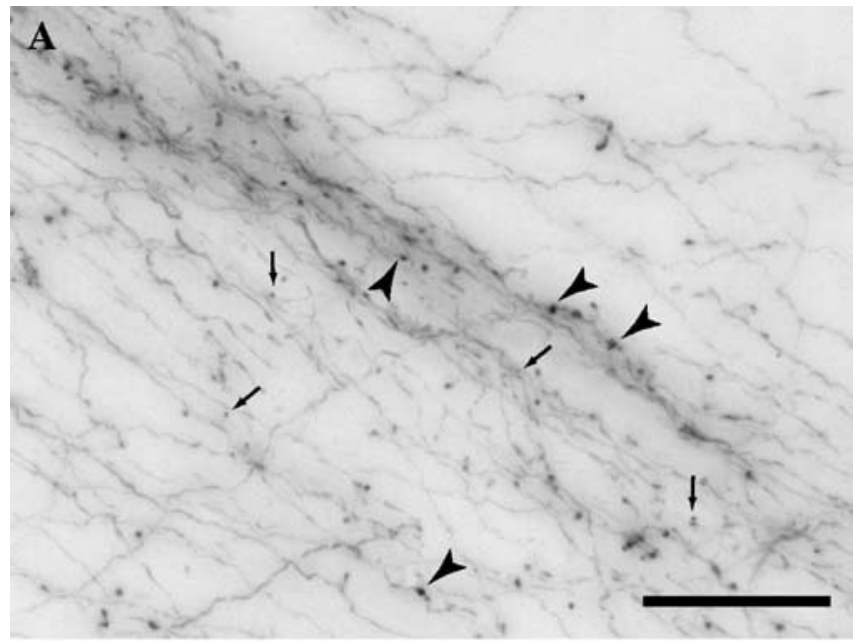

B

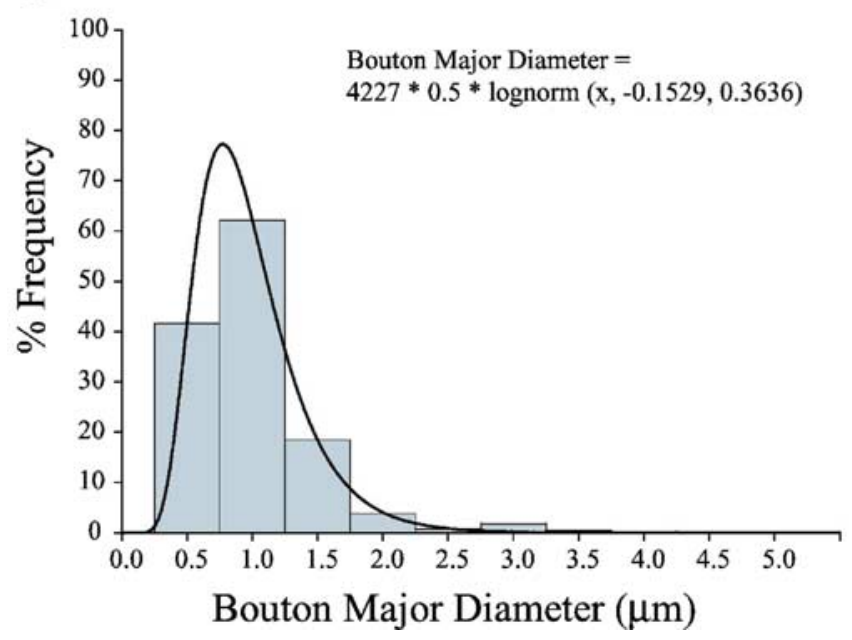

Figure 6. Axonal terminals in TRN were structurally similar regardless of prefrontal cortical origin. Bouton population analysis in area 32 is representative of prefrontal projections. A, Large (black arrowheads) and small (black arrows) boutons in TRN emanating from axons originating in area 32. Scale bar, $50 \mu \mathrm{m} . \boldsymbol{B}$, Bouton frequency distribution in TRN from axons originating in area 32. The population is continuous, best fitted by the lognormal distribution.

consisted of small boutons with round synaptic vesicles and one or no mitochondria (mean volume $\pm \mathrm{SE}, 0.16 \pm 0.02 \mu \mathrm{m}^{3}$; mean area $\left.\pm \mathrm{SE}, 2.07 \pm 0.33 \mu \mathrm{m}^{2}\right)$. The second class consisted of large boutons also with round synaptic vesicles but with two or more mitochondria (mean volume $\pm \mathrm{SE}, 0.41 \pm 0.02 \mu \mathrm{m}^{3}$; mean area $\pm \mathrm{SE}, 3.53 \pm 0.17 \mu \mathrm{m}^{2}$ ). Interestingly, large boutons formed larger synapses (as measured by the areas of the postsynaptic densities) than the small boutons, but the overall ratio of the synapse to bouton area was the same $(0.05 \pm 0.01)$. Comparison of the volumes of labeled boutons with unlabeled presynaptic terminals synapsing on the same or neighboring $\mathrm{PV}^{+}$or $\mathrm{GABA}^{+}$ dendrites showed no significant difference between the two populations.

Some studies in other brain regions and systems have indicated that large boutons synapse primarily on the proximal dendrites and cell bodies, whereas small boutons synapse on distal dendrites (Jones and Powell, 1969; Sato et al., 1997; Liu and Jones, 1999). In the prefrontal pathway to TRN, however, both large and small boutons synapsed on similar dendrites with an average diameter of $1.42 \pm 1.07 \mu \mathrm{m}$, a size that covers both proximal and distal dendrites. 
Similarity of prefrontal terminations in TRN with those in other thalamic nuclei, striatum, and amygdala

To infer the laminar origin of prefrontal corticoreticular terminations, we compared them with a series of other anterogradely labeled bouton populations (at least 4000 per case) in other thalamic nuclei, the caudate, and amygdala, whose laminar origin has been described in detail. This indirect method was necessary because tracer injections exclusively in layers V or VI are difficult to make. Moreover, even if an injection is confined to layer VI, the needle will transect axons from the overlying layer $\mathrm{V}$, which will then be labeled by the tracer. Conversely, an injection limited to cortical layer $\mathrm{V}$ will likely transect and label the dendritic tree of the underlying neurons in layer VI. Single-cell injections in lightly fixed brain slices could provide an alternative and accurate method, but it is very difficult to obtain slices that would include intact axons all the way from the prefrontal cortex to the thalamus of the rhesus monkey brain. Three-dimensional reconstruction of corticothalamic pathways highlights the intricate complexities of such a long pathway and could indicate the feasibility of this approach on a case-by-case basis depending on the site of injection.

Terminals of projections from prefrontal cortex to MD and VA, which originate in layers VI and V, as well as the caudate nucleus and the amygdala (which receive projections mainly from layer $\mathrm{V}$ ) were comparable in size and shape with the bouton population found in TRN from prefrontal cortical projections (Figs.

$10 A-E, 11 A)$. All bouton populations were continuous, covering a wide range of sizes $(0.1 \mu \mathrm{m}<$ diameter $<6 \mu \mathrm{m})$ (Fig. $11 A)$. Cluster analysis indicated that they could include at least two distinct bouton populations, corresponding to a large population of small terminals, and a small population of large boutons $(p<$ 0.00001) (Fig. 11B).

\section{Differences in size of axonal terminations in TRN from prefrontal and sensory cortices}

Previous studies indicated that projections from auditory and other sensory cortices to TRN originate only from layer VI (Rouiller and Welker, 1991, 2000) and terminate exclusively as small boutons. We therefore compared terminations from the temporal auditory (Ts1 and Ts2), visual (TE1), and polymodal (36) association areas in TRN with the prefrontal. The bouton population from the temporal cortices was continuous but differed significantly from all other populations examined, covering a small range of sizes $(0.1 \mu \mathrm{m}<$ diameter $<1.4 \mu \mathrm{m})$ (Figs. $10 \mathrm{~F}$, 11 ) and corresponding to the group of small boutons from prefrontal cortices. These results suggest that prefrontal and sensory corticoreticular projections are structurally different and could have different laminar origins.

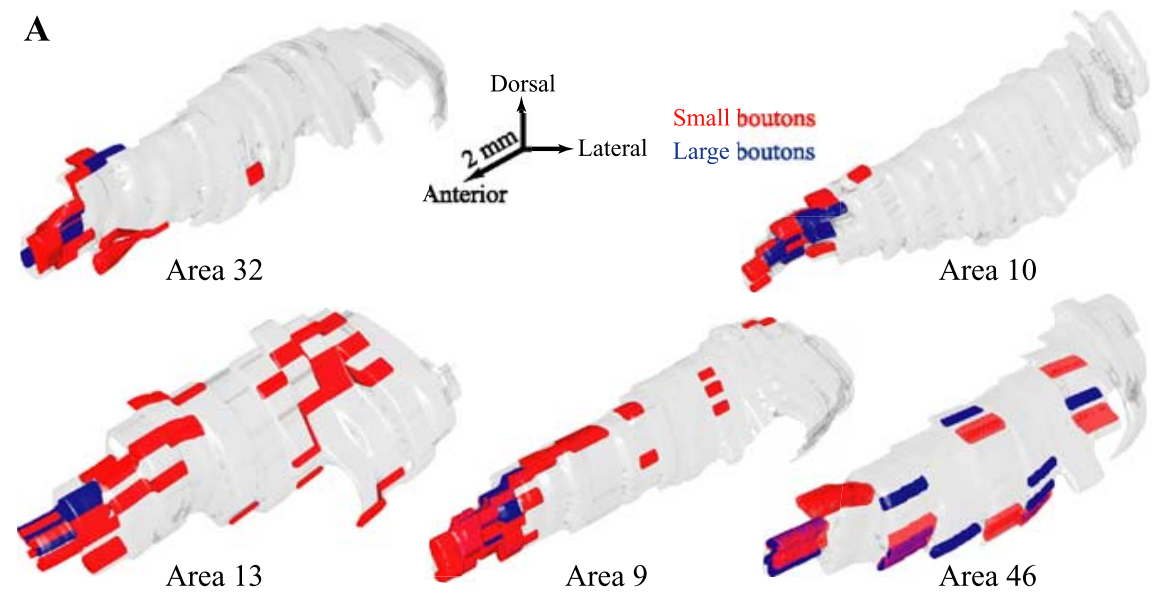

Area 9

Area 46$$
\% \text { Small Boutons }
$$

- $\%$ Small Boutons

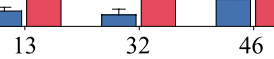

Injection Site
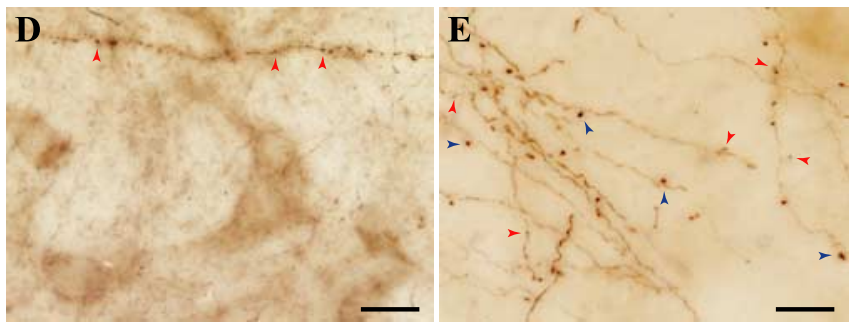

Figure 7. Large and small bouton populations in TRN. $A, 3$ D reconstruction of the topographical distribution of large (blue) and small (red) boutons in TRN from prefrontal axons. $\boldsymbol{B}$, Normalized density (percentage) of large and small boutons in TRN from prefrontal axons. $\boldsymbol{C}-\boldsymbol{E}$, BDA-labeled fibers from prefrontal cortices containing only large (blue arrowheads), only small (red arrowheads), or both kinds of boutons in TRN. Scale bars, $25 \mu \mathrm{m}$.

\section{Methodological controls}

The presence of small and large boutons from prefrontal cortices differed from terminations originating in sensory and motor cortices, which are small. To rule out the possibility that the large boutons seen in TRN belonged to collateral axons from retrogradely labeled neurons in other thalamic nuclei that project to prefrontal cortex and TRN, we used mainly the $10 \mathrm{kDa}$ form of $\mathrm{BDA}, \mathrm{FE}$, or FR, which is optimal for anterograde but not retrograde labeling (Veenman et al., 1992; Reiner et al., 2000). Comparison of the labeled bouton population in these cases and in cases in which a mixture of 3 and $10 \mathrm{kDa} F E$ or FR were used revealed no differences. This finding is consistent with evidence that retrograde labeling over long distances and long survival times is present mainly in the cell body and dendrites and does not enter collateral axons (Wouterlood and Jorritsma-Byham, 1993). Moreover, axons from temporal sensory association cortices terminated exclusively as small boutons in TRN, even when some retrogradely labeled neurons were present in the thalamus.

\section{Discussion}

We present novel evidence on the unique nature of prefrontal cortical projections to TRN (Fig. 12). First, axons from some prefrontal areas terminated broadly in TRN and caudally overlapped with projections from sensory association cortices and 

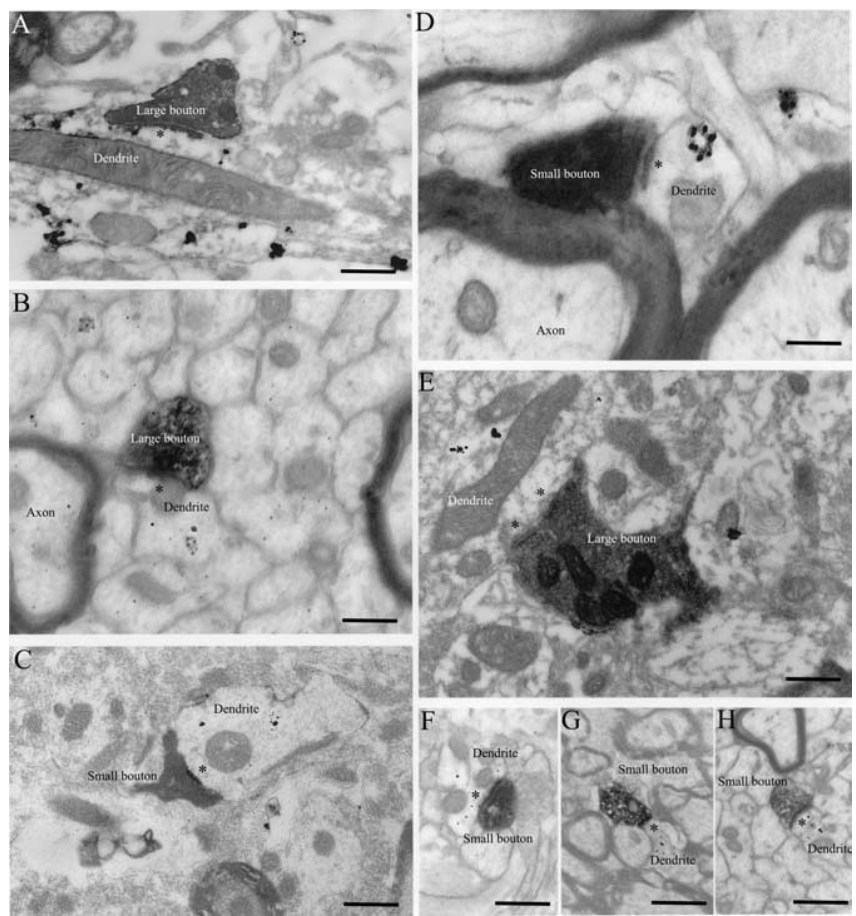

Figure 8. Ultrastructure of large and small boutons in TRN. $A, B$, EM photomicrographs showing large labeled boutons synapsing on $\mathrm{PV}^{+}$dendrites (black dots). C, EM photomicrograph showing a small labeled bouton synapsing on a $\mathrm{PV}^{+}$dendrite. $\boldsymbol{D}$, Small bouton synapsing on a $\mathrm{PV}^{+}$dendrite. $\boldsymbol{E}$, Large bouton synapsing on a $\mathrm{GABA}^{+}$dendrite (black dots). $\boldsymbol{F}-\boldsymbol{H}_{-}$ Small boutons synapsing on $\mathrm{GABA}^{+}$dendrites. Asterisks indicate postsynaptic densities. Dendrites labeled for PV or GABA contain gold particles (black dots). Scale bars: $A-C, E-H, 1 \mu \mathrm{m} ; \boldsymbol{D}$, $0.5 \mu \mathrm{m}$.

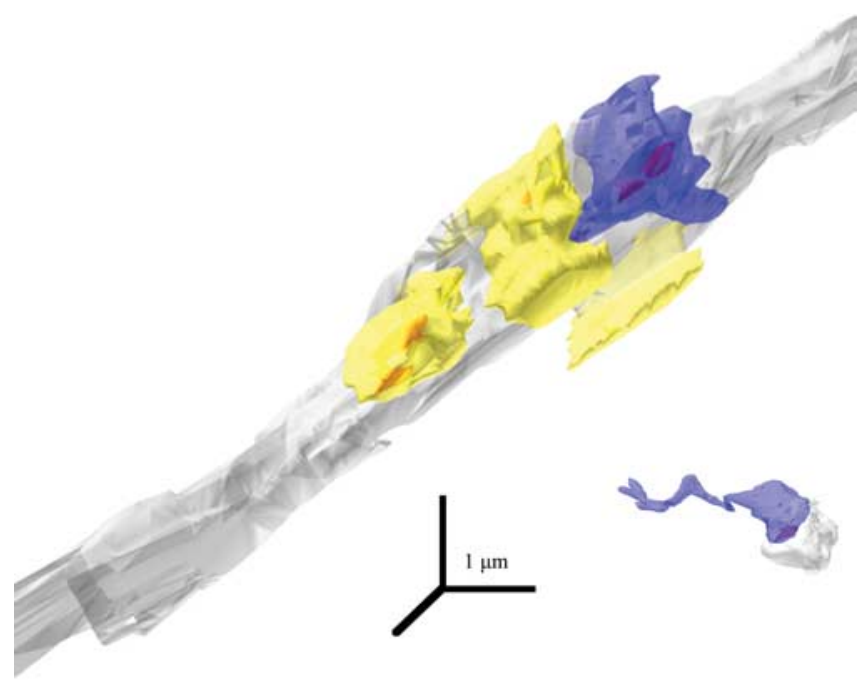

Figure 9. Labeled large and small boutons from prefrontal axons in TRN. 3D reconstruction of the boutons shown in Figure 7, $A$ and $C$, (blue) synapsing (red) on $\mathrm{PV}^{+}$dendrites (gray). Unlabeled boutons (yellow) are included for comparison. The large bouton at the top is at least three times bigger than the small bouton (bottom right).

TRN connections with MD. Second, unlike sensory and motor cortices, prefrontal axons exhibited a dual mode of termination in TRN, as elaborated below.

\section{Topography of cortical projections in TRN: the prefrontal sector}

In rats, cats, rabbits, and primates, cortical and thalamic afferents in TRN are topographically organized (Crabtree and Killackey,
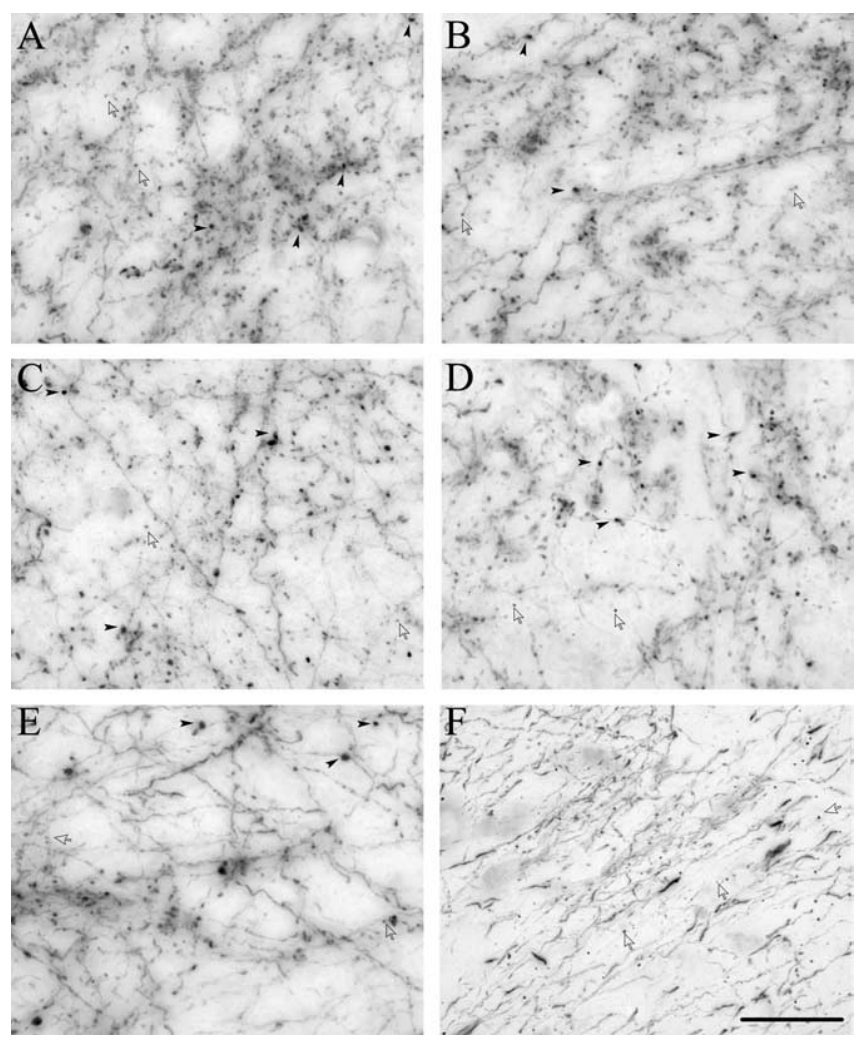

Figure 10. Prefrontal projections to subcortical structures are similar but differ from temporal sensory projections to TRN. Bright-field photomicrographs showing BDA-labeled prefrontal $(\boldsymbol{A}-\boldsymbol{E})$ or temporal $(\boldsymbol{F})$ axons and boutons terminating in various subcortical areas. $\boldsymbol{A}-\boldsymbol{E}$, Terminations from area 32 in MD $(\boldsymbol{A}), \mathrm{VA}(\boldsymbol{B})$, caudate nucleus $(\boldsymbol{C})$, amygdala $(\boldsymbol{D})$, and TRN $(\boldsymbol{E})$. $\boldsymbol{F}$, Terminations in TRN from anterior temporal sensory association areas TE1/Ts1. Note the similarity of all prefrontal terminations, consisting of large boutons (black arrowheads) and small boutons (silhouette arrows) with comparable diameters and surface areas. Terminals from anterior temporal sensory cortices consist solely of small boutons. Scale bar, $25 \mu \mathrm{m}$.

1989; Cicirata et al., 1990; Conley and Diamond, 1990; Cornwall et al., 1990; Crabtree, 1992a,b, 1996, 1998, 1999; Lozsadi, 1994, 1995; Ilinsky et al., 1999). Along the rostrocaudal axis, centroposterior loci in TRN are respectively linked with the somatosensory, visual, and auditory cortices and their associated thalamic nuclei.

In contrast to the detailed information on the central and caudal TRN sectors, little is known about its anterior part, which includes a motor sector, connected with motor cortex and ventrolateral thalamic nuclei, and the rostral pole, associated with the limbic cortex and anterior, midline, and intralaminar thalamic nuclei (Cicirata et al., 1990; Gonzalo-Ruiz and Lieberman, 1995; Lozsadi, 1995; Tai et al., 1995; Kolmac and Mitrofanis, 1997; Xiao and Barbas, 2004). We now provide evidence that the anterior quarter of TRN constitutes the prefrontal sector, overlapping with the motor and limbic sectors. In agreement with previous studies (for review, see Guillery and Harting, 2003), there was considerable overlap in the terminal fields of distinct prefrontal areas. Moreover, the prefrontal TRN sector projects to $\mathrm{MD}, \mathrm{VA}$, and AM, three thalamic nuclei connected with prefrontal cortices (Pinault and Deschenes, 1998; Ilinsky et al., 1999; Xiao and Barbas, 2004).

The most focal projections originated in prefrontal areas 10 and 32 and terminated in the rostral pole of TRN. Interestingly, such focal projection is uncharacteristic for area 32, which has otherwise widespread cortical and subcortical connections (Barbas, 2000). In contrast, terminations from areas 46,13 , and 9 were 

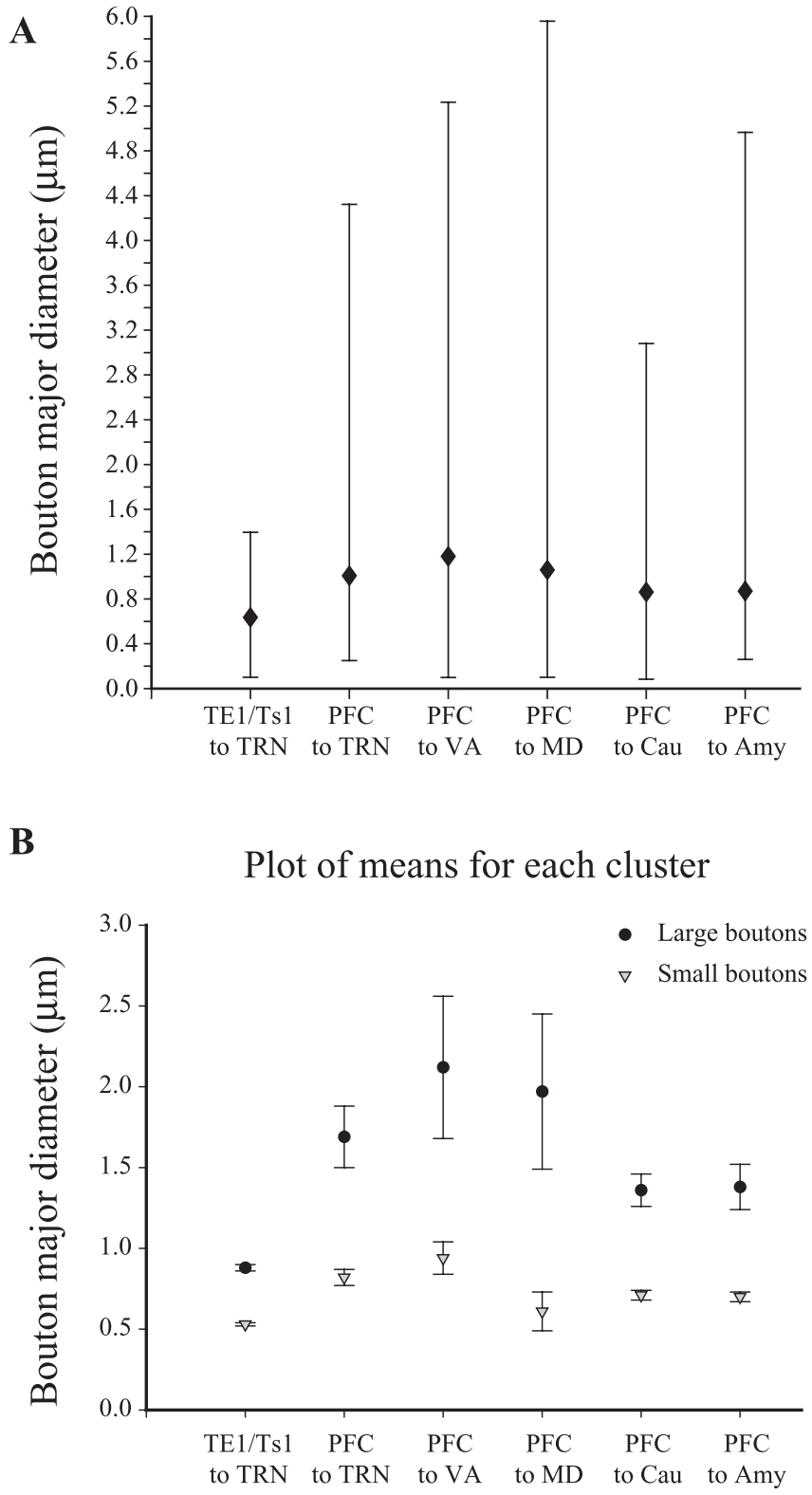

Figure 11. Bouton population analysis of prefrontal and temporal terminations in TRN and subcortical structures. $A$, Mean major diameter of boutons from axons originating in prefrontal cortices or TE/Ts1 and terminating in TRN, VA, MD, caudate, and amygdala. Range of diameters of boutons is also indicated (solid bars). $B$, Cluster analysis of the diameter of boutons terminating in TRN, VA, MD, caudate, and amygdala emanating from prefrontal cortices or TE1/Ts1. The latter are clearly small.

widespread in TRN, extending to its posterior (sensory) sectors. These projections overlapped with terminations from temporal auditory, visual, and polymodal association cortices within the second and third TRN quarters. Areas 46 and 13 exhibited the most extensive overlaps with temporal cortices, followed by area 9. None of the sensory association or polymodal areas studied had such widespread projections, consistent with their topography in other species.

Presynaptic diversity of prefrontal corticoreticular pathways There was also a unique mode of prefrontal terminations in TRN, consisting mostly of small but also a significant proportion of large boutons. In contrast, projections from sensory and motor cortices terminate exclusively as small boutons in TRN and are

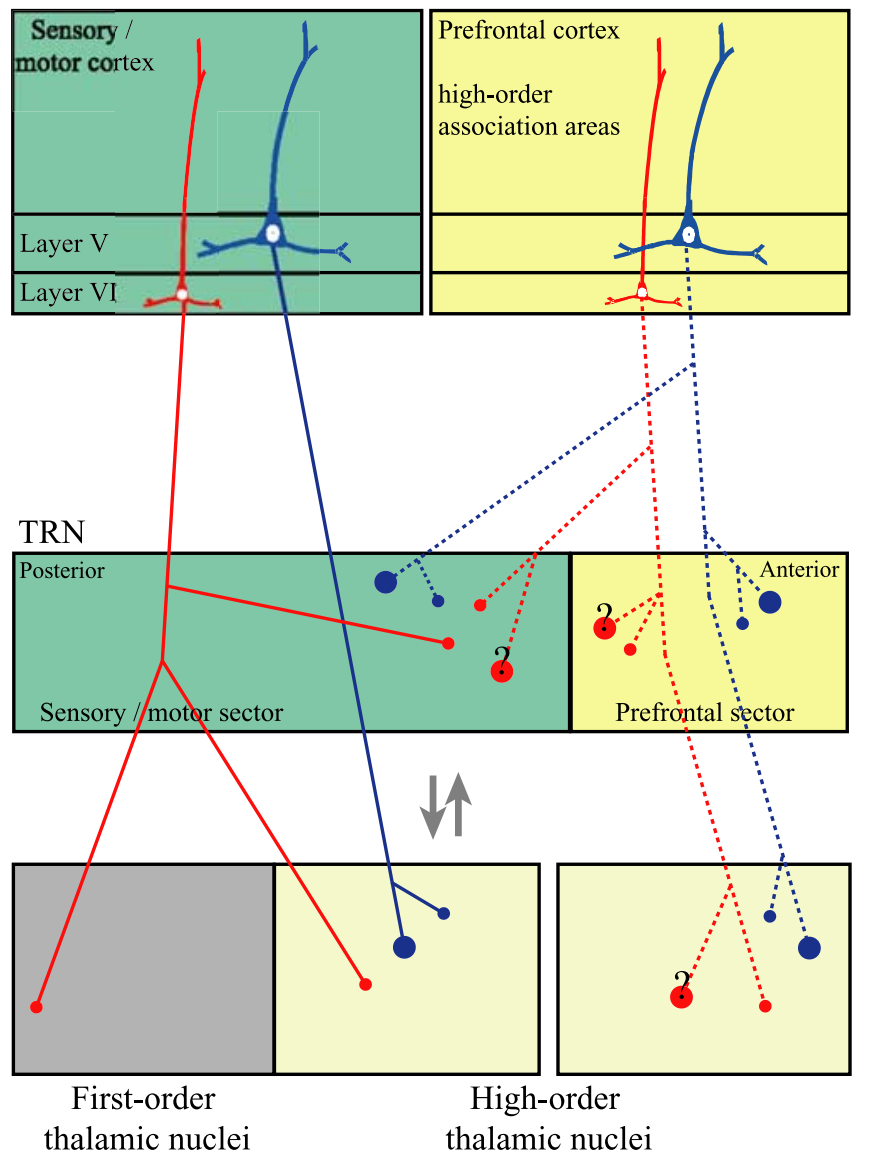

Figure 12. Schematic diagram summarizing the differences of corticothalamic projections issued by prefrontal and sensory/motor cortices. Sensory and motor cortices issue the majority of their thalamic projections from layer $\mathrm{VI}$, which terminate in the thalamus as small boutons (red lines and small red dots) and send collateral branches to TRN. Layer V neurons issue some projections to high-order thalamic nuclei mainly through large boutons, but they do not send collaterals to TRN (blue lines and large blue dots). The prefrontal cortex issues substantial corticothalamic projections from layer $V$, which terminate as large and small boutons in highorder association nuclei of the thalamus. However, it seems that both layers $V$ and $V I$ of the prefrontal cortex send collaterals to TRN, and their terminations are dimorphic (dotted red and blue lines). Axon collaterals from some prefrontal areas extend to central and caudal loci of TRN, in sectors that receive projections from motor and sensory cortices. Question marks on large boutons originating from layer VI neurons highlight the current notion that these neurons terminate exclusively as small boutons, suggesting that the issuance of large terminals is less probable.

thought to originate in layer VI (Guillery et al., 1998; Rouiller et al., 1998; Kakei et al., 2001; Rouiller and Durif, 2004), which is also the predominant pattern of cortical innervation of other thalamic nuclei. Our results confirm and extend these findings by showing that axonal boutons from auditory, visual, and polymodal association cortices were also small. Some corticothalamic neurons are located in layer $\mathrm{V}$, and these are thought to be the source of large boutons in several thalamic nuclei, although not TRN. Consistent with this hypothesis, we found both small and large boutons in prefrontal projections to MD and VA, which originate from layers VI and V (Arikuni et al., 1983; Giguere and Goldman-Rakic, 1988; Schwartz et al., 1991; McFarland and Haber, 2002; Xiao and Barbas, 2004). However, we also found similar populations of small and large prefrontal terminations in the amygdala and caudate, which receive the vast majority of their cortical projections from layer V (Aggleton et al., 1980; Arikuni and Kubota, 1986; Tanaka, 1987; Amaral and Insausti, 1992; Yeterian and Pandya, 1994; McDonald, 1998). Moreover, small and 
large terminals were frequently found along the same axonal branches, suggesting that layer V and/or layer VI corticothalamic neurons can produce both endings. The above findings suggest that the most probable source of large boutons in TRN is layer V, although their origin in layer VI cannot be excluded.

Corticothalamic pathways from layer VI are considered "feedback modulators" of thalamic relay, whereas those from layer $\mathrm{V}$ are thought to be "drivers," similar to the ascending sensory afferents to first-order thalamic nuclei (Guillery and Sherman, 2002). Layer V projections reach high-order thalamic nuclei and are thought to have a role in corticocortical communication, relaying preprocessed information from one area to another cortical area. In this context, prefrontal cortices, unlike other cortices, issue some "driver-like" projections to TRN. Large boutons are more likely to undergo multivesicular release (Rosenmund and Stevens, 1996; Murthy et al., 1997) and could be more efficient in activating TRN, suggested by the linear correlation between number of vesicles and bouton size (Pierce and Lewin, 1994; Shepherd and Harris, 1998; Germuska et al., 2006). Large boutons may be present in highly active networks, consistent with their increased mitochondrial content, which is activity dependent (Thomson, 2000). Reticular neurons are additionally coupled by electrical synapses (Landisman et al., 2002; Landisman and Connors, 2005), suggesting that even a small number of large boutons could initiate widespread activation in TRN and override input from small terminals arising in other cortices. Neighboring reticular neurons also communicate synaptically and thus can inhibit adjacent TRN regions (Zhang and Jones, 2004). As a result, subsets of thalamic neurons could be inhibited and others disinhibited, facilitating discrimination of incoming information.

Small and large boutons from prefrontal cortices may affect differentially the excitability of TRN neurons in the sleep-wake cycle. Glutamatergic cortical afferents, in general, provide the predominance of synaptic inputs in TRN (Liu and Jones, 1999) and likely have a critical role in controlling the firing patterns of thalamocortical relay neurons (Pinault, 2004; Fuentealba and Steriade, 2005). Physiological studies and computational models of reconstructed reticular neurons have shown that the numbers of glutamate receptor subunits are significantly higher in corticoreticular synapses than in other corticothalamic synapses. Moreover, high densities of the low-threshold $\mathrm{Ca}^{2+}$ current in reticular dendrites endow them with exquisite sensitivity to cortical EPSPs when their dendrites are hyperpolarized (Destexhe, 2000; Golshani et al., 2001; Fuentealba and Steriade, 2005). As a result, corticothalamic EPSPs reinforce or evoke bursts in reticular neurons and have a net inhibitory effect on thalamic relay cells, as seen during the early stages of sleep (Steriade et al., 1993b). Conversely, when the dendrites of TRN neurons are depolarized, their activity switches from burst to tonic mode and the cortical influence on relay cells is mostly excitatory, resulting in the desynchronization of thalamocortical oscillations and transition to the alert state. Interestingly, area 46, which had the most widespread projections, was the only area studied that had large terminations in the caudal (sensory) sectors of TRN.

The noradrenergic and cholinergic systems, which have been implicated in the sleep-wake cycle, arousal, and attentional mechanisms (McCormick, 1989; Steriade et al., 1993a; Hasselmo, 1999; Aston-Jones et al., 1999), heavily innervate TRN (Cicirata et al., 1990; Asanuma, 1992; Heckers et al., 1992; Kolmac and Mitrofanis, 1998), and their input could be modulated by the widespread prefrontal projections. These neurotransmitter systems and dopamine also innervate robustly thalamic nuclei connected with prefrontal cortices. A delicate balance of norepineph- rine, acetylcholine, and dopamine is essential for prefrontal cortical function (McCormick et al., 1991; Arnsten and Li, 2005; Sanchez-Gonzalez et al., 2005), and its disruption is implicated in several diseases, including attention deficit disorder and schizophrenia.

\section{Implications of widespread prefrontal projections to TRN on thalamocortical communication}

Prefrontal projections to TRN can regulate the activity of thalamic relay neurons in MD, VA, and AM. In addition, through their widespread projections to TRN, the prefrontal cortices could influence relay cells in other high-order or first-order thalamic nuclei situated beneath the central and posterior TRN sectors. The prefrontal cortex could thus exert indirect control on other cortices or subcortical structures. The unique prefrontalTRN interactions combined with the pacemaker attributes of TRN in the sleep-wake cycle are consistent with the hypothesis that TRN acts as the attentional searchlight of the brain (Crick, 1984). In the rat visual system, for example, a focus of attention is transmitted from the visual cortex to the thalamus, generating a core of excitation surrounded by inhibition initiated by the disynaptic cortico-reticulo-geniculate pathway (Montero, 1999). Approximately half of TRN synapses on anterior and mediodorsal thalamic nuclei in monkeys are with GABAergic neurons, suggesting disinhibition of thalamic relay neurons projecting to prefrontal cortices (Kultas-Ilinsky et al., 1995; Tai et al., 1995). Consistent with this circuit, lesions of TRN increase the incidence of inhibition of thalamocortical projection neurons by local GABAergic neurons (Pinault, 2004; Fuentealba and Steriade, 2005).

The widespread terminations of prefrontal areas 46 and 13 in TRN may help modulate signals conveyed by efferents from temporal sensory association cortices in the same TRN sites. Efferents from area 46 overlap in TRN with projections from inferior temporal cortices associated with visual perception and visual memory (Fuster et al., 1981; Gross, 1994). Area 46 processes detailed sensory information, has a key role in working memory (Goldman-Rakic, 1996; Fuster, 1997; Funahashi and Takeda, 2002), and is connected with premotor cortices for action (Matelli et al., 1986; Barbas and Pandya, 1987). Conversely, area 13 has robust connections with limbic structures and is associated with emotional processing (Barbas, 2000). The interface of prefrontal-sensory association pathways in TRN could have a role in selecting relevant and motivationally significant signals and suppressing distractors. The widespread prefrontoreticular projections may thus contribute to the supervisory and modulatory effects that specific prefrontal areas exert over other cortices.

\section{References}

Aggleton JP, Burton MJ, Passingham RE (1980) Cortical and subcortical afferents to the amygdala of the rhesus monkey (Macaca mulatta). Brain Res 190:347-368.

Amaral DG, Insausti R (1992) Retrograde transport of D- $\left[{ }^{3} \mathrm{H}\right]$-aspartate injected into the monkey amygdaloid complex. Exp Brain Res 88:375-388.

Arikuni T, Kubota K (1986) The organization of prefrontocaudate projections and their laminar origin in the macaque monkey: a retrograde study using HRP-Gel. J Comp Neurol 244:492-510.

Arikuni T, Sakai M, Kubota K (1983) Columnar aggregation of prefrontal and anterior cingulate cortical cells projecting to the thalamic mediodorsal nucleus in the monkey. J Comp Neurol 220:116-125.

Arnsten AF, Li BM (2005) Neurobiology of executive functions: catecholamine influences on prefrontal cortical functions. Biol Psychiatry 57:1377-1384.

Asanuma C (1992) Noradrenergic innervation of the thalamic reticular nu- 
cleus: a light and electron microscopic immunohistochemical study in rats. J Comp Neurol 319:299-311.

Aston-Jones G, Rajkowski J, Cohen J (1999) Role of locus coeruleus in attention and behavioral flexibility. Biol Psychiatry 46:1309-1320.

Barbas H (2000) Connections underlying the synthesis of cognition, memory, and emotion in primate prefrontal cortices. Brain Res Bull 52:319-330.

Barbas H, Pandya DN (1987) Architecture and frontal cortical connections of the premotor cortex (area 6) in the rhesus monkey. J Comp Neurol 256:211-218.

Barbas H, Pandya DN (1989) Architecture and intrinsic connections of the prefrontal cortex in the rhesus monkey. J Comp Neurol 286:353-375.

Barbas H, Saha S, Rempel-Clower N, Ghashghaei T (2003) Serial pathways from primate prefrontal cortex to autonomic areas may influence emotional expression. BMC Neurosci 4:25.

Bourassa J, Pinault D, Deschenes M (1995) Corticothalamic projections from the cortical barrel field to the somatosensory thalamus in rats: a single-fibre study using biocytin as an anterograde tracer. Eur J Neurosci $7: 19-30$.

Cicirata F, Angaut P, Serapide MF, Panto MR (1990) Functional organization of the direct and indirect projection via the reticularis thalami nuclear complex from the motor cortex to the thalamic nucleus ventralis lateralis. Exp Brain Res 79:325-337.

Conley M, Diamond IT (1990) Organization of the visual sector of the thalamic reticular nucleus in Galago. Eur J Neurosci 2:211-226.

Cornwall J, Cooper JD, Phillipson OT (1990) Projections to the rostral reticular thalamic nucleus in the rat. Exp Brain Res 80:157-171.

Crabtree JW (1992a) The somatotopic organization within the rabbit's thalamic reticular nucleus. Eur J Neurosci 4:1343-1351.

Crabtree JW (1992b) The somatotopic organization within the cat's thalamic reticular nucleus. Eur J Neurosci 4:1352-1361.

Crabtree JW (1996) Organization in the somatosensory sector of the cat's thalamic reticular nucleus. J Comp Neurol 366:207-222.

Crabtree JW (1998) Organization in the auditory sector of the cat's thalamic reticular nucleus. J Comp Neurol 390:167-182.

Crabtree JW (1999) Intrathalamic sensory connections mediated by the thalamic reticular nucleus. Cell Mol Life Sci 56:683-700.

Crabtree JW, Killackey HP (1989) The topographic organization and axis of projection within the visual sector of the rabbit's thalamic reticular nucleus. Eur J Neurosci 1:94-109.

Crick F (1984) Function of the thalamic reticular complex: the searchlight hypothesis. Proc Natl Acad Sci USA 81:4586-4590.

Dermon CR, Barbas H (1994) Contralateral thalamic projections predominantly reach transitional cortices in the rhesus monkey. J Comp Neurol 344:508-531.

Destexhe A (2000) Modelling corticothalamic feedback and the gating of the thalamus by the cerebral cortex. J Physiol (Paris) 94:391-410.

Fiala JC (2005) Reconstruct: a free editor for serial section microscopy. J Microsc 218:52-61.

Fiala JC, Harris KM (2001) Cylindrical diameters method for calibrating section thickness in serial electron microscopy. J Microsc 202:468-472.

Fuentealba P, Steriade M (2005) The reticular nucleus revisited: Intrinsic and network properties of a thalamic pacemaker. Prog Neurobiol $75: 125-141$.

Funahashi S, Takeda K (2002) Information processes in the primate prefrontal cortex in relation to working memory processes. Rev Neurosci 13:313-345.

Fuster JM (1997) Network memory. Trends Neurosci 20:451-459.

Fuster JM, Bauer RH, Jervey JP (1981) Effects of cooling inferotemporal cortex on performance of visual memory tasks. Exp Neurol 71:398-409.

Germuska M, Saha S, Fiala JC, Barbas H (2006) Synaptic distinction of laminar specific prefrontal-temporal pathways in primates. Cereb Cortex 16:865-875.

Giguere M, Goldman-Rakic PS (1988) Mediodorsal nucleus: areal, laminar, and tangential distribution of afferents and efferents in the frontal lobe of rhesus monkeys. J Comp Neurol 277:195-213.

Goldman-Rakic PS (1996) Regional and cellular fractionation of working memory. Proc Natl Acad Sci USA 93:13473-13480.

Golshani P, Liu XB, Jones EG (2001) Differences in quantal amplitude reflect GluR4-subunit number at corticothalamic synapses on two populations of thalamic neurons. Proc Natl Acad Sci USA 98:4172-4177.

Gonzalo-Ruiz A, Lieberman AR (1995) Topographic organization of pro- jections from the thalamic reticular nucleus to the anterior thalamic nuclei in the rat. Brain Res Bull 37:17-35.

Gross CG (1994) How inferior temporal cortex became a visual area. Cereb Cortex 5:455-469.

Guillery RW (1995) Anatomical evidence concerning the role of the thalamus in corticocortical communication: a brief review. J Anat 187:583-592.

Guillery RW, Harting JK (2003) Structure and connections of the thalamic reticular nucleus: advancing views over half a century. J Comp Neurol 463:360-371.

Guillery RW, Sherman SM (2002) Thalamic relay functions and their role in corticocortical communication: generalizations from the visual system. Neuron 33:163-175.

Guillery RW, Feig SL, Lozsadi DA (1998) Paying attention to the thalamic reticular nucleus. Trends Neurosci 21:28-32.

Gundersen HJ (1986) Stereology of arbitrary particles. A review of unbiased number and size estimators and the presentation of some new ones, in memory of William R. Thompson. J Microsc 143:3-45.

Hasselmo ME (1999) Neuromodulation: acetylcholine and memory consolidation. Trends Cogn Sci 3:351-359.

Heckers S, Geula C, Mesulam MM (1992) Cholinergic innervation of the human thalamus: dual origin and differential nuclear distribution. J Comp Neurol 325:68-82.

Howard CV, Reed MG (1998) Unbiased stereology, three-dimensional measurement in microscopy. Oxford: BIOS Scientific.

Ilinsky IA, Ambardekar AV, Kultas-Ilinsky K (1999) Organization of projections from the anterior pole of the nucleus reticularis thalami (NRT) to subdivisions of the motor thalamus: light and electron microscopic studies in the rhesus monkey. J Comp Neurol 409:369-384.

Jensen FE, Harris KM (1989) Preservation of neuronal ultrastructure in hippocampal slices using rapid microwave-enhanced fixation. J Neurosci Methods 29:217-230.

Jones EG (1985) The thalamus. New York: Plenum.

Jones EG, Powell TP (1969) An electron microscopic study of the mode of termination of cortico-thalamic fibres within the sensory relay nuclei of the thalamus. Proc R Soc Lond B Biol Sci 172:173-185.

Kakei S, Na J, Shinoda Y (2001) Thalamic terminal morphology and distribution of single corticothalamic axons originating from layers 5 and 6 of the cat motor cortex. J Comp Neurol 437:170-185.

Kievit J, Kuypers HGJM (1975) Subcortical afferents to the frontal lobe in the rhesus monkey studied by means of retrograde horseradish peroxidase transport. Brain Res 85:261-266.

Kievit J, Kuypers HGJM (1977) Organization of the thalamo-cortical connexions to the frontal lobe in the rhesus monkey. Exp Brain Res 29:299-322.

Kolmac CI, Mitrofanis J (1997) Organisation of the reticular thalamic projection to the intralaminar and midline nuclei in rats. J Comp Neurol 377:165-178.

Kolmac CI, Mitrofanis J (1998) Patterns of brainstem projection to the thalamic reticular nucleus. J Comp Neurol 396:531-543.

Kultas-Ilinsky K, Yi H, Ilinsky IA (1995) Nucleus reticularis thalami input to the anterior thalamic nuclei in the monkey: a light and electron microscopic study. Neurosci Lett 186:25-28.

Landisman CE, Connors BW (2005) Long-term modulation of electrical synapses in the mammalian thalamus. Science 310:1809-1813.

Landisman CE, Long MA, Beierlein M, Deans MR, Paul DL, Connors BW (2002) Electrical synapses in the thalamic reticular nucleus. J Neurosci 22:1002-1009.

Liu XB, Jones EG (1999) Predominance of corticothalamic synaptic inputs to thalamic reticular nucleus neurons in the rat. J Comp Neurol 414:67-79.

Lozsadi DA (1994) Organization of cortical afferents to the rostral, limbic sector of the rat thalamic reticular nucleus. J Comp Neurol 341:520-533.

Lozsadi DA (1995) Organization of connections between the thalamic reticular and the anterior thalamic nuclei in the rat. J Comp Neurol 358:233-246.

Matelli M, Camarda R, Glickstein M, Rizzolatti G (1986) Afferent and efferent projections of the inferior area 6 in the macaque monkey. J Comp Neurol 251:281-298.

McCormick DA (1989) Cholinergic and noradrenergic modulation of thalamocortical processing. Trends Neurosci 12:215-221.

McCormick DA, Pape HC, Williamson A (1991) Actions of norepinephrine 
in the cerebral cortex and thalamus: implications for function of the central noradrenergic system. Prog Brain Res 88:293-305.

McDonald AJ (1998) Cortical pathways to the mammalian amygdala. Prog Neurobiol 55:257-332.

McFarland NR, Haber SN (2002) Thalamic relay nuclei of the basal ganglia form both reciprocal and nonreciprocal cortical connections, linking multiple frontal cortical areas. J Neurosci 22:8117-8132.

Montero VM (1999) Amblyopia decreases activation of the corticogeniculate pathway and visual thalamic reticularis in attentive rats: a "focal attention" hypothesis. Neuroscience 91:805-817.

Murthy VN, Sejnowski TJ, Stevens CF (1997) Heterogeneous release properties of visualized individual hippocampal synapses. Neuron 18:599-612.

Ojima H (1994) Terminal morphology and distribution of corticothalamic fibers originating from layers 5 and 6 of cat primary auditory cortex. Cereb Cortex 4:646-663.

Ojima H, Murakami K, Kishi K (1996) Dual termination modes of corticothalamic fibers originating from pyramids of layers 5 and 6 in cat visual cortical area 17. Neurosci Lett 208:57-60.

Olszewski J (1952) The thalamus of the Macaca mulatta. An atlas for use with the stereotaxic instrument. Basel, Switzerland: Karger.

Peters A, Palay SL, Webster HD (1991) The fine structure of the nervous system. Neurons and their supporting cells. New York: Oxford UP.

Pierce JP, Lewin GR (1994) An ultrastructural size principle. Neuroscience 58:441-446.

Pinault D (2004) The thalamic reticular nucleus: structure, function and concept. Brain Res Brain Res Rev 46:1-31.

Pinault D, Deschenes M (1998) Projection and innervation patterns of individual thalamic reticular axons in the thalamus of the adult rat: a threedimensional, graphic, and morphometric analysis. J Comp Neurol 391:180-203.

Reiner A, Veenman CL, Medina L, Jiao Y, Del Mar N, Honig MG (2000) Pathway tracing using biotinylated dextran amines. J Neurosci Methods 103:23-37.

Richmond FJR, Gladdy R, Creasy JL, Ditamura S, Smits E, Thomson DB (1994) Efficacy of seven retrograde tracers, compared in multiplelabelling studies of feline motoneurones. J Neurosci Methods 53:35-46.

Rockland KS (1996) Two types of corticopulvinar terminations: round (type 2) and elongate (type1). J Comp Neurol 368:57-87.

Rosenmund C, Stevens CF (1996) Definition of the readily releasable pool of vesicles at hippocampal synapses. Neuron 16:1197-1207.

Rouiller EM, Durif C (2004) The dual pattern of corticothalamic projection of the primary auditory cortex in macaque monkey. Neurosci Lett 358:49-52.

Rouiller EM, Welker E (1991) Morphology of corticothalamic terminals arising from the auditory cortex of the rat: a Phaseolus vulgarisleucoagglutinin (PHA-L) tracing study. Hear Res 56:179-190.

Rouiller EM, Welker E (2000) A comparative analysis of the morphology of corticothalamic projections in mammals. Brain Res Bull 53:727-741.

Rouiller EM, Tanne J, Moret V, Kermadi I, Boussaoud D, Welker E (1998) Dual morphology and topography of the corticothalamic terminals originating from the primary, supplementary motor, and dorsal premotor cortical areas in macaque monkeys. J Comp Neurol 396:169-185.
Sanchez-Gonzalez MA, Garcia-Cabezas MA, Rico B, Cavada C (2005) The primate thalamus is a key target for brain dopamine. J Neurosci 25:6076-6083.

Sato F, Nakamura Y, Shinoda Y (1997) Serial electron microscopic reconstruction of axon terminals on physiologically identified thalamocortical neurons in the cat ventral lateral nucleus. J Comp Neurol 388:613-631.

Schwartz ML, Dekker JJ, Goldman-Rakic PS (1991) Dual mode of corticothalamic synaptic termination in the mediodorsal nucleus of the rhesus monkey. J Comp Neurol 309:289-304.

Shepherd GM, Harris KM (1998) Three-dimensional structure and composition of CA3 $\rightarrow$ CA1 axons in rat hippocampal slices: implications for presynaptic connectivity and compartmentalization. J Neurosci $18: 8300-8310$.

Steriade M (2005) Sleep, epilepsy and thalamic reticular inhibitory neurons. Trends Neurosci 28:317-324.

Steriade M, Amzica F, Nunez A (1993a) Cholinergic and noradrenergic modulation of the slow (approximately $0.3 \mathrm{~Hz}$ ) oscillation in neocortical cells. J Neurophysiol 70:1385-1400.

Steriade M, McCormick DA, Sejnowski TJ (1993b) Thalamocortical oscillations in the sleeping and aroused brain. Science 262:679-685.

Steriade M, Jones EG, McCormick DA (1997) Thalamus: organisation and function. Oxford: Elsevier.

Tai Y, Yi H, Ilinsky IA, Kultas-Ilinsky K (1995) Nucleus reticularis thalami connections with the mediodorsal thalamic nucleus: a light and electron microscopic study in the monkey. Brain Res Bull 38:475-488.

Tanaka Jr D (1987) Differential laminar distribution of corticostriatal neurons in the prefrontal and pericruciate gyri of the dog. J Neurosci 7:4095-4106.

Thomson AM (2000) Molecular frequency filters at central synapses. Prog Neurobiol 62:159-196.

Veenman CL, Reiner A, Honig MG (1992) Biotinylated dextran amine as an anterograde tracer for single- and double-labeling studies. J Neurosci Methods 41:239-254.

Walker AE (1940) A cytoarchitectural study of the prefrontal area of the macaque monkey. J Comp Neurol 73:59-86.

Wouterlood FG, Jorritsma-Byham B (1993) The anterograde neuroanatomical tracer biotinylated dextran- amine: comparison with the tracer Phaseolus vulgaris-leucoagglutinin in preparations for electron microscopy. J Neurosci Methods 48:75-87.

Xiao D, Barbas H (2002) Pathways for emotions and memory. II. Afferent input to the anterior thalamic nuclei from prefrontal, temporal, hypothalamic areas and the basal ganglia in the rhesus monkey. Thalamus Related Systems 2:33-48.

Xiao D, Barbas H (2004) Circuits through prefrontal cortex, basal ganglia, and ventral anterior nucleus map pathways beyond motor control. Thalamus Related Systems 2:325-343.

Yeterian EH, Pandya DN (1994) Laminar origin of striatal and thalamic projections of the prefrontal cortex in rhesus monkeys. Exp Brain Res 99:383-398.

Zhang L, Jones EG (2004) Corticothalamic inhibition in the thalamic reticular nucleus. J Neurophysiol 91:759-766. 